\title{
On best proximity points for pseudocontractions in the intermediate sense for non-cyclic and cyclic self-mappings in metric spaces
}

Manuel De la Sen

"Correspondence:

manuel.delasen@ehu.es Institute of Research and

Development of Processes, University of the Basque Country, Campus of Leioa (Bizkaia), P.O. Box 644, Bilbao, 48080, Spain

\begin{abstract}
This paper discusses a more general contractive condition for a class of extended 2-cyclic self-mappings on the union of a finite number of subsets of a metric space which are allowed to have a finite number of successive images in the same subsets of its domain. If the space is uniformly convex and the subsets are nonempty, closed and convex, then all the iterations converge to a unique closed limiting finite sequence, which contains the best proximity points of adjacent subsets, and reduce to a unique fixed point if all such subsets intersect.
\end{abstract}

\section{Introduction}

Strict pseudocontractive mappings and pseudocontractive mappings in the intermediate sense formulated in the framework of Hilbert spaces have received a certain attention in the last years concerning their convergence properties and the existence of fixed points. See, for instance, [1-4] and references therein. Results about the existence of a fixed point are discussed in those papers. On the other hand, important attention has been paid during the last decades to the study of the convergence properties of distances in cyclic contractive self-mappings on $p$ subsets $A_{i} \subset X$ of a metric space $(X, d)$, or a Banach space $(X,\|\|)$. The cyclic self-mappings under study have been of standard contractive or weakly contractive types and of Meir-Keeler type. The convergence of sequences to fixed points and best proximity points of the involved sets has been investigated in the last years. See, for instance, [5-20] and references therein. It has to be noticed that every nonexpansive mapping $[21,22]$ is a 0 -strict pseudocontraction and also that strict pseudocontractions in the intermediate sense are asymptotically nonexpansive [2]. The uniqueness of the best proximity points to which all the sequences of iterations converge is proven in [6] for the extension of the contractive principle for cyclic self-mappings in either uniformly convex Banach spaces (then being strictly convex and reflexive [23]) or in reflexive Banach spaces [13]. The $p$ subsets $A_{i} \subset X$ of the metric space $(X, d)$, or the Banach space $(X,\|\|)$, where the cyclic self-mappings are defined, are supposed to be nonempty, convex and closed. If the involved subsets have nonempty intersections, then all best proximity points coincide, with a unique fixed point being allocated in the intersection of all the subsets, and framework can be simply given on complete metric spaces. The research in [6] is centered

(c) 2013 De la Sen; licensee Springer. This is an Open Access article distributed under the terms of the Creative Commons Attribution License (http://creativecommons.org/licenses/by/2.0), which permits unrestricted use, distribution, and reproduction in any medium, provided the original work is properly cited. 
on the case of the 2-cyclic self-mapping being defined on the union of two subsets of the metric space. Those results are extended in [7] for Meir-Keeler cyclic contraction maps and, in general, with the $p(\geq 2)$-cyclic self-mapping $T: \bigcup_{i \in \bar{p}} A_{i} \rightarrow \bigcup_{i \in \bar{p}} A_{i}$ defined on any number of subsets of the metric space with $\bar{p}:=\{1,2, \ldots, p\}$. Other recent research which has been performed in the field of cyclic maps is related to the introduction and discussion of the so-called cyclic representation of a set $M$, as the union of a set of nonempty sets as $M=\bigcup_{i=1}^{m} M_{i}$, with respect to an operator $f: M \rightarrow M$ [14]. Subsequently, cyclic representations have been used in [15] to investigate operators from $M$ to $M$ which are cyclic $\varphi$-contractions, where $\varphi: \mathbf{R}_{0+} \rightarrow \mathbf{R}_{0+}$ is a given comparison function, $M \subset X$ and $(X, d)$ is a metric space. The above cyclic representation has also been used in [16] to prove the existence of a fixed point for a self-mapping defined on a complete metric space which satisfies a cyclic weak $\varphi$-contraction. In [18], a characterization of best proximity points is studied for individual and pairs of non-self-mappings $S, T: A \rightarrow B$, where $A$ and $B$ are nonempty subsets of a metric space. The existence of common fixed points of self-mappings is investigated in [24] for a class of nonlinear integral equations, while fixed point theory is investigated in locally convex spaces and non-convex sets in [25-28]. More recently, the existence and uniqueness of best proximity points of more general cyclic contractions have been investigated in $[29,30]$ and a study of best proximity points for generalized proximal contractions, a concept referred to non-self-mappings, has been proposed and reported in detail in [31]. Also, the study and characterization of best proximity points for cyclic weaker Meir-Keeler contractions have been performed in [32] and recent contributions on the study of best proximity and proximal points can be found in [33-38] and references therein. In general, best proximity points do not fulfill the usual 'best proximity' condition $x=S x=T x$ under this framework. However, best proximity points are proven to jointly globally optimize the mappings from $x$ to the distances $d(x, T x)$ and $d(x, S x)$. Furthermore, a class of cyclic $\varphi$-contractions, which contains the cyclic contraction maps as a subclass, has been proposed in [18] in order to investigate the convergence and existence results of best proximity points in reflexive Banach spaces completing previous related results in [6]. Also, the existence and uniqueness of best proximity points of cyclic $\varphi$-contractive selfmappings in reflexive Banach spaces have been investigated in [19]. This paper is devoted to the convergence properties and the existence of fixed points of a generalized version of pseudocontractive, strict pseudocontractive and asymptotically pseudocontractive in the intermediate sense in the more general framework of metric spaces. The case of 2-cyclic pseudocontractive self-mappings is also considered. The combination of constants defining the contraction may be different on each of the subsets and only the product of all the constants is requested to be less than unity. It is assumed that the considered self-mapping can perform a number of iterations on each of the subsets before transferring its image to the next adjacent subset of the 2-cyclic self-mapping. The existence of a unique closed finite limiting sequence on any sequence of iterations from any initial point in the union of the subsets is proven if $X$ is a uniformly convex Banach space and all the subsets of $X$ are nonempty, convex and closed. Such a limiting sequence is of size $q \geq p$ (with the inequality being strict if there is at least one iteration with image in the same subset as its domain), where $p$ of its elements (all of them if $q=p$ ) are best proximity points between adjacent subsets. In the case that all the subsets $A_{i} \subset X$ intersect, the above limit sequence reduces to a unique fixed point allocated within the intersection of all such subsets. 


\section{Asymptotic contractions and pseudocontractions in the intermediate sense in metric spaces}

If $H$ is a real Hilbert space with an inner product $\langle\cdot, \cdot\rangle$ and a norm $\|\cdot\|$ and $A$ is a nonempty closed convex subset of $H$, then $T: A \rightarrow A$ is said to be an asymptotically $\beta$-strictly pseudocontractive self-mapping in the intermediate sense for some $\beta \in[0,1)$ if

$$
\limsup _{n \rightarrow \infty} \sup _{x, y \in A}\left(\left\|T^{n} x-T^{n} y\right\|^{2}-\alpha_{n}\|x-y\|^{2}-\beta\left\|\left(I-T^{n}\right) x-\left(I-T^{n}\right) y\right\|^{2}\right) \leq 0
$$

for some sequence $\left\{\alpha_{n}\right\} \subset[1, \infty), \alpha_{n} \rightarrow 1$ as $n \rightarrow \infty[1-4,23]$. Such a concept was firstly introduced in [1]. If (2.1) holds for $\beta=1$, then $T: A \rightarrow A$ is said to be an asymptotically pseudocontractive self-mapping in the intermediate sense. Finally, if $\alpha_{n} \rightarrow \alpha \in[0,1)$ as $n \rightarrow \infty$, then $T: A \rightarrow A$ is asymptotically $\beta$-strictly contractive in the intermediate sense, respectively, asymptotically contractive in the intermediate sense if $\beta=1$. If (2.1) is changed to the stronger condition

$$
\begin{aligned}
& \left(\left\|T^{n} x-T^{n} y\right\|^{2}-\alpha_{n}\|x-y\|^{2}-\beta\left\|\left(I-T^{n}\right) x-\left(I-T^{n}\right) y\right\|^{2}\right) \\
& \quad \leq 0 ; \quad \forall x, y \in A, n \in \mathbf{N}
\end{aligned}
$$

then the above concepts translate into $T: A \rightarrow A$ being an asymptotically $\beta$-strictly pseudocontractive self-mapping, an asymptotically pseudocontractive self-mapping and asymptotically contractive one, respectively. Note that (2.1) is equivalent to

$$
\begin{aligned}
& \left\|T^{n} x-T^{n} y\right\|^{2} \\
& \quad \leq \alpha_{n}\|x-y\|^{2}+\beta\left\|\left(I-T^{n}\right) x-\left(I-T^{n}\right) y\right\|^{2}+\xi_{n} ; \quad \forall x, y \in A, \forall n \in \mathbf{N}
\end{aligned}
$$

or, equivalently,

$$
\begin{aligned}
& \left\langle T^{n} x-T^{n} y, x-y\right\rangle \\
& \quad \leq \frac{1}{2 \beta}\left[\left(\alpha_{n}+\beta\right)\|x-y\|^{2}+(\beta-1)\left\|T^{n} x-T^{n} y\right\|^{2}+\xi_{n}\right] ; \quad \forall x, y \in A, n \in \mathbf{N},
\end{aligned}
$$

where

$$
\begin{aligned}
\xi_{n}:= & \max \left\{0, \sup _{x, y \in A}\left(\left\|T^{n} x-T^{n} y\right\|^{2}-\alpha_{n}\|x-y\|^{2}\right.\right. \\
& \left.\left.-\beta\left\|\left(I-T^{n}\right) x-\left(I-T^{n}\right) y\right\|^{2}\right)\right\} ; \quad \forall n \in \mathbf{N} .
\end{aligned}
$$

Note that the high-right-hand-side term $\left\|\left(I-T^{n}\right) x-\left(I-T^{n}\right) y\right\|^{2}$ of $(2.3)$ is expanded as follows for any $x, y \in A$ :

$$
\begin{aligned}
\| x & -y\left\|^{2}+\right\| T^{n} x-T^{n} y\left\|^{2}-2\right\| x-y\|\| T^{n} x-T^{n} y \| \\
& \leq\left\|\left(I-T^{n}\right) x-\left(I-T^{n}\right) y\right\|^{2} \leq\left\langle x-T^{n} x, y-\left.T^{n} y\right|^{2}=\left\langle x-y, T^{n} x-\left.T^{n} y\right|^{2}\right.\right. \\
& =\|x-y\|^{2}+\left\|T^{n} x-T^{n} y\right\|^{2}+2\left\langle T^{n} x-T^{n} y, x-y\right\rangle \\
& =\left\langle x-y, T^{n} x-T^{n} y\right\rangle\left\langle x-y, T^{n} x-T^{n} y\right\rangle
\end{aligned}
$$




$$
\begin{aligned}
& \leq\|x-y\|^{2}+\left\|T^{n} x-T^{n} y\right\|^{2}+2\left|\left\langle T^{n} x-T^{n} y, x-y\right\rangle\right| \\
& \leq\|x-y\|^{2}+\left\|T^{n} x-T^{n} y\right\|^{2}+2\|x-y\|\left\|T^{n} x-T^{n} y\right\| .
\end{aligned}
$$

The objective of this paper is to discuss the various pseudocontractive in the intermediate sense concepts in the framework of metric spaces endowed with a homogeneous and translation-invariant metric and also to generalize them to the $\beta$-parameter to eventually be replaced with a sequence $\left\{\beta_{n}\right\}$ in $(0,1)$. Now, if instead of a real Hilbert space $H$ endowed with an inner product $\langle\cdot, \cdot\rangle$ and a norm $\|\cdot\|$, we deal with any generic Banach space $(X,\|\cdot\|)$, then its norm induces a homogeneous and translation invariant metric $d: X \times X \rightarrow \mathbf{R}_{0_{+}}$defined by $d(x, y)=d(x-y, 0)=\|x-y\|^{1 / 2} ; \forall x, y \in A$ so that (2.6) takes the form

$$
\begin{aligned}
& d^{2}(x, y)+d^{2}\left(T^{n} x, T^{n} y\right)-2 d(x, y) d\left(T^{n} x, T^{n} y\right) \\
& \quad \leq\left\|\left(I-T^{n}\right) x-\left(I-T^{n}\right) y\right\|^{2}=d^{2}\left(x-y-\left(T^{n} x-T^{n} y\right), 0\right)=d^{2}\left(x-y, T^{n} x-T^{n} y\right) \\
& \quad \leq\left(d(x-y, 0)+d\left(T^{n} x-T^{n} y, 0\right)\right)^{2}=\left(d(x, y)+d\left(T^{n} x, T^{n} y\right)\right)^{2} \\
& \quad=d^{2}(x, y)+d^{2}\left(T^{n} x, T^{n} y\right)+2 d(x, y) d\left(T^{n} x, T^{n} y\right) ; \quad \forall x, y \in A .
\end{aligned}
$$

Define

$$
\begin{aligned}
\mu_{n}(x, y):= & \min \left(\rho \in[-1,1]: d^{2}\left(x-y, T^{n} x-T^{n} y\right) \leq d^{2}(x, y)+d^{2}\left(T^{n} x, T^{n} y\right)\right. \\
& \left.+2 \rho d(x, y) d\left(T^{n} x, T^{n} y\right)\right) ; \quad \forall x, y \in A, \forall n \in \mathbf{N},
\end{aligned}
$$

which exists since it follows from (2.7), since the metric is homogeneous and translationinvariant, that

$$
\begin{aligned}
\{1\} \subset & \left\{\rho \in \mathbf{R}:\left\|\left(I-T^{n}\right) x-\left(I-T^{n}\right) y\right\|^{2}\right. \\
& \left.\leq d^{2}(x, y)+d^{2}\left(T^{n} x, T^{n} y\right)+2 \rho d(x, y) d\left(T^{n} x, T^{n} y\right)\right\}(\neq \varnothing) .
\end{aligned}
$$

The following result holds related to the discussion (2.7)-(2.9) in metric spaces.

Theorem 2.1 Let $(X, d)$ be a metric space and consider a self-mapping $T: X \rightarrow X$. Assume that the following constraint holds:

$$
\begin{aligned}
& d^{2}\left(T^{n} x, T^{n} y\right) \\
& \quad \leq \alpha_{n}(x, y) d^{2}(x, y)+\beta_{n}(x, y)\left(d^{2}(x, y)+d^{2}\left(T^{n} x, T^{n} y\right)\right) \\
& \quad+2 \mu_{n}(x, y) \beta_{n}(x, y) d(x, y) d\left(T^{n} x, T^{n} y\right)+\xi_{n}(x, y) ; \quad \forall x, y \in X, \forall n \in \mathbf{N}
\end{aligned}
$$

with

$$
\begin{aligned}
\xi_{n}= & \xi_{n}(x, y) \\
:= & \max \left(0,\left(1-\beta_{n}(x, y)\right) d^{2}\left(T^{n} x, T^{n} y\right)-\left(\alpha_{n}(x, y)+\beta_{n}(x, y)\right) d^{2}(x, y)\right. \\
& \left.-2 \mu_{n}(x, y) \beta_{n}(x, y) d(x, y) d\left(T^{n} x, T^{n} y\right)\right) \rightarrow 0 ; \quad \forall x, y \in X \text { as } n \rightarrow \infty
\end{aligned}
$$


for some parameterizing bounded real sequences $\left\{\alpha_{n}(x, y)\right\},\left\{\beta_{n}(x, y)\right\}$ and $\left\{\mu_{n}(x, y)\right\}$ of general terms $\alpha_{n}=\alpha_{n}(x, y), \beta_{n}=\beta_{n}(x, y), \mu_{n}=\mu_{n}(x, y)$ satisfying the following constraints:

$$
\begin{aligned}
& {\left[\left(\mu_{n}(x, y) \in\left[-\frac{\alpha_{n}(x, y)+\beta_{n}(x, y)}{2 \beta_{n}(x, y)}, \frac{1-\alpha_{n}(x, y)-2 \beta_{n}(x, y)}{2 \beta_{n}(x, y)}\right]\right) \wedge\left(\beta_{n}(x, y)<1\right)\right]} \\
& \quad \vee\left[\left(\mu_{n}(x, y)<-\frac{\alpha_{n}(x, y)+\beta_{n}(x, y)}{2 \beta_{n}(x, y)}\right) \wedge\left(\beta_{n}(x, y)>1\right) \Leftrightarrow \xi_{n}(x, y)=0\right] \\
& \quad \vee\left[\mu_{n}(x, y) \in\left[\frac{1-\alpha_{n}(x, y)-2 \beta_{n}(x, y)}{2 \beta_{n}(x, y)}, \frac{1-\beta_{n}(x, y)}{2 \beta_{n}(x, y)}\right)\right] ; \quad \forall x, y \in X, \forall n \in \mathbf{N}
\end{aligned}
$$

with $\lim \sup _{n \rightarrow \infty}\left[\beta_{n}(x, y) \max \left(1,1+2 \mu_{n}(x, y)\right)\right]<1$ and, furthermore, the following condition is satisfied:

$$
\left(\mu_{n}(x, y)-\frac{1-\alpha_{n}(x, y)-2 \beta_{n}(x, y)}{2 \beta_{n}(x, y)}\right) \rightarrow 0 ; \quad \forall x, y \in X \text { as } n \rightarrow \infty
$$

if and only if $\alpha_{n}+2 \beta_{n}\left(1+\mu_{n}\right) \rightarrow 1 ; \forall x, y \in X$ as $n \rightarrow \infty$.

Then the following properties hold:

(i) $\exists \lim _{n \rightarrow \infty} d\left(T^{n} x, T^{n} y\right) \leq d(x, y)$ for any $x, y \in X$ so that $T: X \rightarrow X$ is asymptotically nonexpansive.

(ii) Let $(X, d)$ be complete, $d: X \times X \rightarrow \mathbf{R}_{0+}$ be, in addition, a translation-invariant homogeneous norm and let $(X,\|\|) \equiv(X, d)$, with \|\| being the metric-induced norm from $d: X \times X \rightarrow \mathbf{R}_{0+}$, be a uniformly convex Banach space. Assume also that $T: X \rightarrow X$ is continuous. Then any sequence $\left\{T^{n} x\right\} ; \forall x \in A$ is bounded and convergent to some point $z_{x}=z_{x}(x) \in C$, being in general dependent on $x$, in some nonempty bounded, closed and convex subset $C$ of $A$, where $A$ is any nonempty bounded subset of $X$. Also, $d\left(T^{n} x, T^{n+m} x\right)$ is bounded; $\forall n, m \in \mathbf{N}, \lim _{n \rightarrow \infty} d\left(T^{n} x, T^{n+m} x\right)=0 ; \forall x \in A, \forall m \in \mathbf{N}$ and $z_{x}=z_{x}(x)=T z_{x} \in C$ is a fixed point of the restricted self-mapping $T: C \rightarrow C ; \forall x \in A$. Furthermore,

$$
\lim _{n \rightarrow \infty}\left(d^{2}\left(T^{n+1} x, T^{n+1} y\right)-d^{2}\left(T^{n} x, T^{n} y\right)\right)=0 ; \quad \forall x, y \in A .
$$

Proof Consider two possibilities for the constraint (2.10), subject to (2.11), to hold for each given $x, y \in X$ and $n \in \mathbf{N}$ as follows:

(A) $d\left(T^{n} x, T^{n} y\right) \leq d(x, y)$ for any $x, y \in X, n \in \mathbf{N}$. Then one gets from (2.10)

$$
\begin{aligned}
& d^{2}\left(T^{n} x, T^{n} y\right) \leq\left(\alpha_{n}+\beta_{n}\right) d^{2}(x, y)+\beta_{n} d^{2}\left(T^{n} x, T^{n} y\right)+2 \mu_{n} \beta_{n} d^{2}(x, y)+\xi_{n} \\
& \quad \Rightarrow \quad d\left(T^{n} x, T^{n} y\right) \leq k_{a n} d^{2}(x, y)+\frac{\xi_{n}}{1-\beta_{n}} ;
\end{aligned}
$$

$\forall x, y \in A, \forall n \in \mathbf{N}$, where

$$
k_{a n}=k_{a n}(x, y)=\frac{\alpha_{n}+\beta_{n}\left(1+2 \mu_{n}\right)}{1-\beta_{n}} \rightarrow 1 ; \quad \forall x, y \in X \text { as } n \rightarrow \infty,
$$

which holds from (2.12)-(2.13) if $\lim _{\sup } \operatorname{sum}_{n \rightarrow \infty} \beta_{n}(x, y)<1$ since

$$
\left(\mu_{n}(x, y)-\frac{1-\alpha_{n}(x, y)-2 \beta_{n}(x, y)}{2 \beta_{n}(x, y)}\right) \rightarrow 0 ;
$$


$\forall x, y \in X$ as $n \rightarrow \infty$ in (2.13) is equivalent to (2.16). Note that $0 \leq k_{a n} \leq 1$ is ensured either with $\min \left(\alpha_{n}+\beta_{n}\left(1+2 \mu_{n}\right), 1-\beta_{n}\right) \geq 0$ or with $\max \left(\alpha_{n}+\beta_{n}\left(1+2 \mu_{n}\right), 1-\beta_{n}\right) \leq 0$ if

$$
\begin{aligned}
& {\left[\left(\mu_{n}(x, y) \in\left[-\frac{\alpha_{n}(x, y)+\beta_{n}(x, y)}{2 \beta_{n}(x, y)}, \frac{1-\alpha_{n}(x, y)-2 \beta_{n}(x, y)}{2 \beta_{n}(x, y)}\right]\right) \wedge\left(\beta_{n}(x, y) \in(0,1)\right)\right]} \\
& \quad \vee\left[\left(\mu_{n}(x, y)<\frac{1-\alpha_{n}(x, y)-\beta_{n}(x, y)}{2 \beta_{n}(x, y)}\right) \wedge\left(\beta_{n}(x, y) \geq 1\right)\right] .
\end{aligned}
$$

However, $\beta_{n}>1$ with $\xi_{n}>0$ has to be excluded because of the unboundedness or nonnegativity of the second right-hand-side term of (2.15).

(B) $d\left(T^{n} x, T^{n} y\right) \geq d(x, y)$ for some $x, y \in X, n \in \mathbf{N}$. Then one gets from (2.10)

$$
\begin{aligned}
& d\left(T^{n} x, T^{n} y\right)^{2} \leq\left(\alpha_{n}+\beta_{n}\right) d^{2}(x, y)+\beta_{n} d^{2}\left(T^{n} x, T^{n} y\right)+2 \mu_{n} \beta_{n} d^{2}\left(T^{n} x, T^{n} y\right)+\xi_{n} \\
& \Rightarrow \quad d\left(T^{n} x, T^{n} y\right)^{2} \leq k_{b n} d^{2}(x, y)+\frac{\xi_{n}}{1-\beta_{n}\left(1+2 \mu_{n}\right)}
\end{aligned}
$$

where

$$
k_{b n}=k_{b n}(x, y)=\frac{\alpha_{n}+\beta_{n}}{1-\beta_{n}\left(1+2 \mu_{n}\right)} \rightarrow 1 \quad \text { as } n \rightarrow \infty,
$$

which holds from (2.12) and $k_{b n} \geq 1$ if $\limsup _{n \rightarrow \infty}\left[\beta_{n}(x, y) \max \left(1,1+2 \mu_{n}(x, y)\right)\right]<1$, and

$$
\mu_{n}(x, y) \in\left[\frac{1-\alpha_{n}(x, y)-2 \beta_{n}(x, y)}{2 \beta_{n}(x, y)}, \frac{1-\beta_{n}(x, y)}{2 \beta_{n}(x, y)}\right) .
$$

Thus, (2.15)-(2.16), with the second option in the logic disjunction being true if and only if $\xi_{n}=0$ together with (2.18)-(2.20), are equivalent to (2.12)-(2.13) by taking $k_{n}=k_{n}(x, y)$ to be either $k_{a n}$ or $k_{b n}$ for each $n \in \mathbf{N}$. It then follows that $\exists \lim \sup _{n \rightarrow \infty}\left(d\left(T^{n} x, T^{n} y\right)-d(x, y)\right) \leq 0$; $\forall x, y \in X$ from (2.15)-(2.19) since $0 \leq k_{n}=k_{n}(x, y) \leq 1$ and $k_{n}(x, y) \rightarrow 1 ; \forall x, y \in X$ as $n \rightarrow \infty$. Thus, $T: X \rightarrow X$ is asymptotically nonexpansive. Thus, Property (i) has been proven. Property (ii) is proven as follows. Consider the metric-induced norm \|| || equivalent to the translation-invariant homogeneous metric $d: X \times X \rightarrow \mathbf{R}_{0_{+}}$. Such a norm exists since the metric is homogeneous and translation-invariant so that norm and metric are formally equivalent. Rename $A_{0} \equiv A$ and define a sequence of subsets $A_{j}:=\left\{T^{j} x: x \in A_{0}\right\}$ of $X$. From Property (i), $\left\{d\left(T^{n} x, T^{n} y\right)\right\}$ is bounded; $\forall x, y \in X$ if $d(x, y)$ is finite, since it is bounded for any finite $n \in \mathbf{N}$ and, furthermore, it has a finite limit as $n \rightarrow \infty$. Thus, all the collections of subsets $\bigcup_{i=1}^{k} A_{i} ; \forall k \in \mathbf{N}$ are bounded since $A_{0}$ is bounded. Define the set $C=C\left(A_{0}\right):=\operatorname{cl}\left[\right.$ convex $\left.\left(\bigcup_{i=1}^{\infty} A_{k}\right)\right]$ which is nonempty bounded, closed and convex by construction. Since $(X, d)$ is complete, $(X,\|\|) \equiv(X, d)$ is a uniformly convex Banach space and $T: C \rightarrow C$ is asymptotically nonexpansive from Property (i), then it has a fixed point $z=T z \in C[1,23]$. Since the restricted self-mapping $T: C \rightarrow C$ is also continuous, one gets from Property (i)

$$
\exists \lim _{n \rightarrow \infty} d\left(T^{n} x, T^{n} z\right)=\lim _{n \rightarrow \infty} d\left(T^{n} x, z\right)=d\left(\lim _{n \rightarrow \infty} T^{n} x, z\right) \leq d(x, z)<\infty ; \quad \forall x \in A .
$$

Then any sequence $\left\{T^{n} x\right\}$ is convergent (otherwise, the above limit would not exist contradicting Property (i)), and then bounded in $C ; \forall x \in A$. This also implies $d\left(T^{n} x, T^{n+m} x\right)$ is 
bounded; $\forall x \in A, \forall n, m \in \mathbf{N}$ and $\lim _{n \rightarrow \infty} d\left(T^{n} x, T^{n+m} x\right)=0 ; \forall x \in A, \forall m \in \mathbf{N}$. This implies also $T^{n} x \rightarrow z_{x}(x)$ as $n \rightarrow \infty ; \forall x \in A$ such that $z_{x}(x)=T z_{x} ; \forall x \in A$ which is then a fixed point of $T: C \rightarrow C$ (otherwise, the above property $\lim _{n \rightarrow \infty} d\left(T^{n} x, T^{n+m} x\right)=0 ; \forall x \in A, \forall m \in \mathbf{N}$ would be contradicted). Hence, Property (ii) is proven.

First of all, note that Property (ii) of Theorem 2.1 applies to a uniformly convex space which is also a complete metric space. Since the metric is homogeneous and translationinvariant, a norm can be induced by such a metric. Alternatively, the property could be established on any uniformly convex Banach space by taking a norm-induced metric which always exists. Conceptually similar arguments are used in later parallel results throughout the paper. Note that the proof of Theorem 2.1(i) has two parts: Case (A) refers to an asymptotically nonexpansive self-mapping which is contractive for any number of finite iteration steps and Case (B) refers to an asymptotically nonexpansive self-mapping which is allowed to be expansive for a finite number of iteration steps. It has to be pointed out concerning such a Theorem 2.1(ii) that the given conditions guarantee the existence of at least a fixed point but not its uniqueness. Therefore, the proof is outlined with the existence of a $z \in \operatorname{Fix}(T \mid C)$ for any nonempty, bounded and closed subset $A$ of $X$. Note that the set $C$, being in general dependent on the initial set $A$, is bounded, convex and closed by construction while any taken nonempty set of initial conditions $A \subset X$ is not required to be convex. However, the property that all the sequences converge to fixed points opens two potential possibilities depending on particular extra restrictions on the self-mapping $T: C \rightarrow C$, namely: (1) the fixed point is not unique so that $z_{x} \equiv z$ for any $x \in A$ (and any $A$ in $X$ ) so that some set $\operatorname{Fix}(T \mid C)$ for some $C=C(A) \subset X$ contains more than one point. In other words, $d^{2}\left(T^{n} x, T^{n} y\right) \rightarrow 0$ as $n \rightarrow \infty ; \forall x, y \in A$ has not been proven although it is true that $\lim _{n \rightarrow \infty}\left(d^{2}\left(T^{n+1} x, T^{n+1} y\right)-d^{2}\left(T^{n} x, T^{n} y\right)\right)=0 ; \forall x, y \in A$; (2) there is only a fixed point in $X$. The following result extends Theorem 2.1 for a modification of the asymptotically nonexpansive condition (2.10).

Theorem 2.2 Let $(X, d)$ be a metric space and consider the self-mapping $T: X \rightarrow X$. Assume that the constraint below holds:

$$
\begin{aligned}
d^{2}\left(T^{n} x, T^{n} y\right) \leq & \alpha_{n}(x, y) d^{2}(x, y)+\beta_{n}(x, y)\left(d^{2}(x, y)+d^{2}\left(T^{n} x, T^{n} y\right)\right) \\
& +2 \mu_{n}(x, y) \beta_{n}(x, y) d^{2}\left(T^{n} x, T^{n} y\right)+\xi_{n}(x, y) ; \quad \forall x, y \in X, \forall n \in \mathbf{N}
\end{aligned}
$$

with

$$
\begin{aligned}
\xi_{n}= & \xi_{n}(x, y) \\
:= & \max \left(0,\left(1-\beta_{n}(x, y)\right) d^{2}\left(T^{n} x, T^{n} y\right)-\left(\alpha_{n}(x, y)+\beta_{n}(x, y)\right) d^{2}(x, y)\right. \\
& \left.-2 \mu_{n}(x, y) \beta_{n}(x, y) d^{2}\left(T^{n} x, T^{n} y\right)\right) \rightarrow 0 ; \quad \forall x, y \in X \text { as } n \rightarrow \infty
\end{aligned}
$$

for some parameterizing real sequences $\alpha_{n}=\alpha_{n}(x, y), \beta_{n}=\beta_{n}(x, y)$ and $\mu_{n}=\mu_{n}(x, y)$ satisfying, for any $n \in \mathbf{N}$,

$$
\begin{aligned}
& \left\{\alpha_{n}(x, y)\right\} \subset[0, \infty), \quad\left\{\mu_{n}(x, y)\right\} \subset\left[-1, \frac{1-\beta_{n}(x, y)}{2 \beta_{n}(x, y)}\right), \\
& \left\{\beta_{n}(x, y)\right\} \subset[0,1] ; \quad \forall x, y \in X, \forall n \in \mathbf{N} .
\end{aligned}
$$


Then the following properties hold:

(i) $\exists \lim _{n \rightarrow \infty} d\left(T^{n} x, T^{n} y\right) \leq d(x, y)$ so that $T: X \rightarrow X$ is asymptotically nonexpansive, and then $\exists \lim _{n \rightarrow \infty} d\left(T^{n} x, T^{n} y\right) \leq d(x, y) ; \forall x, y \in X$ if

$$
\begin{aligned}
& \frac{\alpha_{n}(x, y)+\beta_{n}(x, y)}{1-\beta_{n}(x, y)\left(1+2 \mu_{n}(x, y)\right)} \geq 1 \\
& \quad \Leftrightarrow \quad \mu_{n}(x, y) \in\left[\frac{1-\alpha_{n}(x, y)-2 \beta_{n}(x, y)}{2 \beta_{n}(x, y)}, \frac{1-\beta_{n}(x, y)}{2 \beta_{n}(x, y)}\right) ; \quad \forall x, y \in X, \forall n \in \mathbf{N}
\end{aligned}
$$

and the following limit exists:

$$
\alpha_{n}(x, y)+2 \beta_{n}(x, y)\left(1+\mu_{n}(x, y)\right) \rightarrow 1 ; \quad \forall x, y \in X \text { as } n \rightarrow \infty .
$$

(ii) Property (ii) of Theorem 2.1 if $(X, d)$ is complete and $(X,\|\|) \equiv(X, d)$ is a uniformly convex Banach space under the metric-induced norm \|\| .

Sketch of the proof Property (i) follows in the same way as the proof of Property (i) of Theorem 2.1 for Case (B). Using proving arguments similar to those used to prove Theorem 2.1, one proves Property (ii).

The relevant part in Theorem 2.1 being of usefulness concerning the asymptotic pseudocontractions in the intermediate sense and the asymptotic strict contractions in the intermediate sense relies on Case (B) in the proof of Property (i) with the sequence of constants $k_{n}(x, y) \geq 1 ; \forall x, y \in X, \forall n \in \mathbf{N}$ and $k_{n}(x, y) \rightarrow 1$; as $n \rightarrow \infty, \forall x, y \in X$. The concepts of an asymptotic pseudocontraction and an asymptotic strict pseudocontraction in the intermediate sense motivated in Theorem 2.1 by (2.7)-(2.9), under the asymptotically nonexpansive constraints (2.10) subject to (2.11) and in Theorem 2.2 by (2.22) subject to (2.23) are revisited as follows in the context of metric spaces.

Definition 2.3 Assume that $(X, d)$ is a complete metric space with $d: X \times X \rightarrow \mathbf{R}_{0+}$ being a homogeneous translation-invariant metric. Thus, $T: A \rightarrow A$ is asymptotically $\beta$-strictly pseudocontractive in the intermediate sense if

$$
\limsup _{n \rightarrow \infty}\left(\left(1-\beta_{n}\left(1+2 \mu_{n}\right)\right) d^{2}\left(T^{n} x, T^{n} y\right)-\left(\alpha_{n}+\beta_{n}\right) d^{2}(x, y)\right) \leq 0 ; \quad \forall x, y \in A
$$

for $\beta_{n}=\beta \in[0,1) ; \forall n \in \mathbf{N}$ and some real sequences $\left\{\alpha_{n}\right\},\left\{\mu_{n}\right\}$ being, in general, dependent on the initial points, i.e., $\alpha_{n}=\alpha_{n}(x, y), \mu_{n}=\mu_{n}(x, y)$ and

$$
\begin{aligned}
& \left\{\mu_{n}\right\} \subset\left[-1, \frac{1-\beta}{2 \beta}\right) \text { and }\left\{\alpha_{n}\right\} \subset[1, \infty) ; \\
& \forall n \in \mathbf{N}, \alpha_{n} \rightarrow 1 \text { and } \mu_{n} \rightarrow-1 \text { as } n \rightarrow \infty ; \forall x, y \in A, \forall n \in \mathbf{N} .
\end{aligned}
$$

Definition 2.4 $T: A \rightarrow A$ is asymptotically pseudocontractive in the intermediate sense if $(2.30)$ holds with $\left\{\mu_{n}\right\} \subset\left[-1, \frac{1-\beta_{n}}{2 \beta_{n}}\right),\left\{\beta_{n}\right\} \subset[0,1],\left\{\alpha_{n}\right\} \subset[1, \infty), \alpha_{n} \rightarrow 1, \beta_{n} \rightarrow 1, \mu_{n} \rightarrow-1$ as $n \rightarrow \infty$ and the remaining conditions as in Definition 2.3 with $\alpha_{n}=\alpha_{n}(x, y), \beta_{n}=\beta_{n}(x, y)$ and $\mu_{n}=\mu_{n}(x, y)$. 
Definition 2.5 $T: A \rightarrow A$ is asymptotically $\beta$-strictly contractive in the intermediate sense if $\alpha_{n} \in[0, \infty), \beta_{n}=\beta \in[0,1), \mu_{n} \in\left[-1, \frac{1-\beta}{2 \beta}\right) ; \forall n \in \mathbf{N}, \mu_{n} \rightarrow \mu \in\left[-1, \frac{1-\beta}{2 \beta} \min \left(1, \frac{1}{\alpha+\beta}\right)\right)$, $\alpha_{n} \rightarrow \alpha \in[0,1)$ as $n \rightarrow \infty$, in Definition 2.3 with $\alpha_{n}=\alpha_{n}(x, y), \mu_{n}=\mu_{n}(x, y)$.

Definition 2.6 $T: A \rightarrow A$ is asymptotically contractive in the intermediate sense if $\alpha_{n} \in$ $[0, \infty),\left\{\beta_{n}\right\} \subset[0,1), \mu_{n} \in\left[-1, \frac{1-\beta_{n}}{2 \beta_{n}}\right) ; \forall n \in \mathbf{N}, \mu_{n} \rightarrow \mu \in\left[-1,-\frac{1+\alpha}{2}\right), \alpha_{n} \rightarrow \alpha \in[0,1)$, and $\beta_{n} \rightarrow \beta=1$ as $n \rightarrow \infty$ in Definition 2.3 with $\alpha_{n}=\alpha_{n}(x, y), \beta_{n}=\beta_{n}(x, y)$ and $\mu_{n}=\mu_{n}(x, y)$.

Remark 2.7 Note that Definitions 2.3-2.5 lead to direct interpretations of their role in the convergence properties under the constraint (2.22), subject to (2.23), by noting the following:

(1) If $T: A \rightarrow A$ is asymptotically $\beta$-strictly pseudocontractive in the intermediate sense (Definition 2.3), then the real sequence $\left\{k_{1 n}\right\}$ of asymptotically nonexpansive constants has a general term $k_{1 n}:=\left(\frac{\alpha_{n}+\beta}{1-\beta\left(1+2 \mu_{n}\right)}\right)^{1 / 2} \in\left[\left(\frac{\alpha_{n}+\beta}{1+\beta}\right)^{1 / 2}, \infty\right) \subset[1, \infty) ; \forall n \in \mathbf{N}$, and it converges to a limit $k_{1}=1$ since $\xi_{n} \rightarrow 0$ and $\alpha_{n} \rightarrow 1$ as $n \rightarrow \infty ; \forall x, y \in A$ from (2.22) since $\mu_{n} \rightarrow-1$ from (2.27). Then $T: A \rightarrow A$ is trivially asymptotically nonexpansive as expected.

(2) If $T: A \rightarrow A$ is asymptotically pseudocontractive in the intermediate sense (Definition 2.4), then the sequence $\left\{k_{2 n}\right\}$ of asymptotically nonexpansive constants has the general term: $k_{2 n}:=\left(\frac{\alpha_{n}+\beta_{n}}{1-\beta_{n}\left(1+2 \mu_{n}\right)}\right)^{1 / 2} \in\left[\left(\frac{\alpha_{n}+\beta_{n}}{1+\beta_{n}}\right)^{1 / 2}, \infty\right) \subset[1, \infty) ; \forall n \in \mathbf{N}$, and it converges to a limit $k_{2}=1$ since $\alpha_{n} \rightarrow 1, \beta_{n} \rightarrow 1$ as $n \rightarrow \infty$. Then $T: A \rightarrow A$ is also trivially asymptotically nonexpansive as expected. Since $\alpha_{n} \geq 1$, note that $\beta_{n}>\beta \Rightarrow k_{2 n}>k_{1 n}$ and $\beta_{n}<\beta \Rightarrow k_{2 n}<k_{1 n}$ for any $n \in \mathbf{N}$, while $k_{1 n} \rightarrow 1, k_{2 n} \rightarrow 1$ as $n \rightarrow \infty$ since $\xi_{n} \rightarrow 0$ as $n \rightarrow \infty ; \forall x, y \in A$ from (2.22)-(2.23).

(3) If $T: A \rightarrow A$ is asymptotically $\beta$-strictly contractive in the intermediate sense (Definition 2.5), then the sequence of asymptotically contractive constants is defined by $k_{3 n}:=\left(\frac{\alpha_{n}+\beta}{1-\beta\left(1+2 \mu_{n}\right)}\right)^{1 / 2} \in\left[\left(\frac{\alpha_{n}+\beta}{1+\beta}\right)^{1 / 2}, \infty\right) \subset\left[\left(\frac{\beta}{1+\beta}\right)^{1 / 2}, \infty\right) ; \forall n \in \mathbf{N}$ and $k_{3 n} \rightarrow k_{3}=\left(\frac{\alpha+\beta}{1-\beta(1+2 \mu)}\right)^{1 / 2} \in[0,1)$ as $n \rightarrow \infty$ for any $\mu \in\left[-1, \frac{1-\alpha-2 \beta}{2 \beta}\right)$ such that $\mu_{n} \rightarrow \mu$ as $n \rightarrow \infty$, since $\alpha+2 \beta(1+\mu)<1$. Then $T: A \rightarrow A$ is an asymptotically strict contraction as expected since $\xi_{n} \rightarrow 0$ as $n \rightarrow \infty ; \forall x, y \in A$ from (2.22)-(2.23). Note that the asymptotic convergence rate is arbitrarily fast as $\alpha$ and $\beta$ are arbitrarily close to zero, since $k_{3}=O(\alpha+\beta)=o(\alpha+\beta)=o(\max (\sqrt{\alpha}, \sqrt{\beta}))$ becomes also arbitrarily close to zero, and $k_{3}^{2} \leq K(\alpha+\beta)$ with $K=K(\beta, \mu)=\frac{1}{1-\beta(1+2 \mu)} \in(0, \infty)$.

(4) If $T: A \rightarrow A$ is asymptotically contractive in the intermediate sense (Definition 2.6), then the sequence of asymptotically contractive constants is defined by

$$
k_{4 n}:=\left(\frac{\alpha_{n}+\beta_{n}}{1-\beta_{n}\left(1+2 \mu_{n}\right)}\right)^{1 / 2} \in\left[\left(\frac{\alpha_{n}+\beta_{n}}{1+\beta_{n}}\right)^{1 / 2}, \infty\right) \subset\left[\left(\frac{\beta_{n}}{1+\beta_{n}}\right)^{1 / 2}, \infty\right) ; \quad \forall n \in \mathbf{N}
$$

with $\beta_{n} \rightarrow \beta=1$ and $k_{4 n} \rightarrow k_{4}=\left(\frac{1+\alpha}{2|\mu|}\right)^{1 / 2} \in\left[\frac{1}{2}, 1\right)$ as $n \rightarrow \infty$ for some $\mu \in\left[-1,-\frac{1+\alpha}{2}\right)$ since $\mu<0$ with $|\mu|>\frac{1+\alpha}{2}$ so that $k_{4} \in\left[\left(\frac{1+\alpha}{2}\right)^{1 / 2}, 1\right)$. Then $T: A \rightarrow A$ is an asymptotically strict contraction as expected since $\xi_{n} \rightarrow 0$ as $n \rightarrow \infty ; \forall x, y \in A$ from (2.23). Note that $k_{3}=k_{4}$ if $\mu<0$ and $|\mu|=\frac{1+\alpha}{2 \alpha}$ and $k_{4}^{2} \geq \frac{1}{2}+o(\alpha) \geq \frac{1}{2}$. Note also that $k_{3}<k_{4}$ if $\mu \leq 0$ and $|\mu|<\frac{1+\alpha}{2 \alpha}$, while $k_{3} \geq k_{4}$ if $\mu \leq 0$ and $|\mu| \geq \frac{1+\alpha}{2 \alpha}$. In the first case, the convergence to fixed points (see Theorem 2.8 below) is guaranteed to be asymptotically faster if the self-mapping is asymptotically $\beta$-strictly contractive in the intermediate sense than if it is just asymptotically contractive in the 
intermediate sense if $\beta_{n}>\beta, n \in \mathbf{N}$. Note also that if the sequences $\left\{\alpha_{n}\right\}$ and $\left\{\mu_{n}\right\}$ are identical in both cases, then $k_{3 n}<k_{4 n}$ for any $n \in \mathbf{N}$ such that $\beta_{n}>\beta$ and $k_{3 n} \geq k_{4 n}$ for any $n \in \mathbf{N}$ such that $\beta_{n} \leq \beta$.

(5) The above considerations could also be applied to Theorem 2.1 for the case $d\left(T^{n} x, T^{n} y\right) \geq d(x, y)$ (Case (B) in the proof of Property (i)) being asymptotically nonexpansive for the asymptotically nonexpansive condition (2.10) subject to (2.11).

The subsequent result, being supported by Theorem 2.2, relies on the concepts of asymptotically contractive and pseudocontractive self-mappings in the intermediate sense. Therefore, it is assumed that $\left\{\alpha_{n}(x, y)\right\} \subset[1, \infty)$.

Theorem 2.8 Let $(X, d)$ be a complete metric space endowed with a homogeneous translation-invariant metric $d: X \times X \rightarrow \mathbf{R}_{0_{+}}$and consider the self-mapping $T: X \rightarrow X$. Assume that $(X,\|\|) \equiv(X, d)$ is a uniformly convex Banach space endowed with a metricinduced norm \|| $\|$ from the metric $d: X \times X \rightarrow \mathbf{R}_{0_{+}}$. Assume that the asymptotically nonexpansive condition (2.22), subject to (2.23), holds for some parameterizing real sequences $\alpha_{n}=\alpha_{n}(x, y), \beta_{n}=\beta_{n}(x, y)$ and $\mu_{n}=\mu_{n}(x, y)$ satisfying, for any $n \in \mathbf{N}$,

$$
\begin{aligned}
& \left\{\alpha_{n}(x, y)\right\} \subset[1, \infty), \quad\left\{\mu_{n}(x, y)\right\} \subset\left[-1, \frac{1-\beta_{n}(x, y)}{2 \beta_{n}(x, y)}\right), \\
& \left\{\beta_{n}(x, y)\right\} \subset[0, \beta) \subset[0,1]
\end{aligned}
$$

$\forall x, y \in X, \forall n \in \mathbf{N}$. Then $\exists \lim _{n \rightarrow \infty} d\left(T^{n} x, T^{n} y\right) \leq d(x, y)$ for any $x, y \in X$ satisfying the conditions

$$
\begin{aligned}
& \frac{\alpha_{n}(x, y)+\beta_{n}(x, y)}{1-\beta_{n}(x, y)\left(1+2 \mu_{n}(x, y) d(x, y)\right)} \geq 1 ; \\
& \alpha_{n}(x, y)+2 \beta_{n}(x, y)\left(1+\mu_{n}(x, y)\right) \rightarrow 1 ; \quad \forall x, y \in X \text { as } n \rightarrow \infty .
\end{aligned}
$$

Furthermore, the following properties hold:

(i) $T: C \rightarrow C$ is asymptotically $\beta$-strictly pseudocontractive in the intermediate sense for some nonempty, bounded, closed and convex set $C=C(A) \subset X$ and any given nonempty, bounded and closed subset $A \subset X$ of initial conditions if (2.29) hold with $0 \leq \beta_{n}=\beta<1$, $\left\{\mu_{n}\right\} \subset\left[-1, \frac{1-\beta}{2 \beta}\right),\left\{\alpha_{n}\right\} \subset[1, \infty), \alpha_{n} \rightarrow 1$ and $\mu_{n} \rightarrow-1$ as $n \rightarrow \infty ; \forall x, y \in A, \forall n \in \mathbf{N}$. Also, $T: C \rightarrow C$ has a fixed point for any such set $C$ if $T: X \rightarrow X$ is continuous.

(ii) $T: C \rightarrow C$ is asymptotically pseudocontractive in the intermediate sense for some nonempty, bounded, closed and convex set $C=C(A) \subset X$ and any given nonempty, bounded and closed subset $A \subset X$ of initial conditions if (2.29) hold with $\left\{\beta_{n}\right\} \subset[0,1],\left\{\mu_{n}\right\} \subset$ $\left[-1, \frac{1-\beta_{n}}{2 \beta_{n}}\right),\left\{\alpha_{n}\right\} \subset[1, \infty), \beta_{n} \rightarrow 1, \alpha_{n} \rightarrow 1$ and $\mu_{n} \rightarrow-1$ as $n \rightarrow \infty ; \forall x, y \in A, \forall n \in \mathbf{N}$. Also, $T: C \rightarrow C$ has a fixed point for any such set $C$ if $T: X \rightarrow X$ is continuous.

(iii) If (2.29) hold with $\alpha_{n} \in[0, \infty), \beta_{n}=\beta \in[0,1), \mu_{n} \in\left[-1, \frac{1-\beta}{2 \beta}\right), \mu_{n} \rightarrow \mu \in\left[-1, \frac{1-\alpha-2 \beta}{2 \beta}\right)$; $\forall n \in \mathbf{N}$ and $\alpha_{n} \rightarrow \alpha \in[0,1)$ as $n \rightarrow \infty$, then $T: X \rightarrow X$ is asymptotically $\beta$-strictly contractive in the intermediate sense. Also, $T: X \rightarrow X$ has a unique fixed point.

(iv) If (2.29) hold with $\alpha_{n} \in[0, \infty),\left\{\beta_{n}\right\} \subset[0,1), \mu_{n} \in\left[-1, \frac{1-\beta_{n}}{2 \beta_{n}}\right), \mu_{n} \rightarrow \mu \in\left[-1,-\frac{1+\alpha}{2}\right)$; $\forall n \in \mathbf{N}, \beta_{n} \rightarrow 1$ and $\alpha_{n} \rightarrow \alpha \in[0,1)$ as $n \rightarrow \infty$, then $T: X \rightarrow X$ is asymptotically strictly contractive in the intermediate sense. Also, $T: X \rightarrow X$ has a unique fixed point. 
Proof (i) It follows from Definition 2.3 and the fact that Theorem 2.2 holds under the particular nonexpansive condition (2.22), subject to (2.23), so that $T: A \rightarrow A$ is asymptotically nonexpansive (see Remark 2.7(1)). Property (ii) follows in a similar way from Definition 2.4 (see Remark 2.7(2)). Properties (iii)-(iv) follow from Theorem 2.2 and Definitions 2.5-2.6 implying also that the asymptotically nonexpansive self-mapping $T: X \rightarrow X$ is also a strict contraction, then continuous with a unique fixed point, since $\alpha+2 \beta(1+\mu)<1$ (see Remark 2.7(3)) and $\mu<0$ with $|\mu|>\frac{1+\alpha}{2}$ (see Remark 2.7(4)), respectively. (The above properties could also be got from Theorem 2.1 for Case (B) of the proof of Theorem 2.1(ii) see Remark 2.7(5).)

Example 2.9 Consider the time-varying $p$ th order nonlinear discrete dynamic system

$$
x_{k+n}=T^{n} x_{k} \equiv x_{k+1}+F_{n}\left(x_{k}\right)\left(x_{k+1}-x_{k}\right)+\eta_{n}\left(x_{k}\right) ; \quad x_{1} \in A_{1} \subset \mathbf{R}^{p}, \forall k \in \mathbf{N} ;
$$

$\forall k, n \in \mathbf{N}$ for some given nonempty bounded set $A_{1}$, where $\left\{F_{n k}\right\}$ is a $\mathbf{R}^{p \times p}$ matrix sequence of elements $F_{n k}: \mathbf{R}^{p} \rightarrow \mathbf{R}^{p \times p}$ with $F_{n k}=F_{n}\left(x_{k}\right)$ and $\eta_{n k}: \mathbf{R}^{p} \rightarrow \mathbf{R}^{p}$ with $\eta_{n k}=\eta_{n}\left(x_{k}\right) ; \forall k \in \mathbf{N}$, and $T: \mathbf{R}^{p} \rightarrow \mathbf{R}^{p}$ defines the state-sequence trajectory solution $\left\{x_{k}\left(x_{1}, x_{2}\right)\right\}$. Equation (2.13) requires the consistency constraint $F_{1} \neq 1$ to calculate $x_{2}$. However, other discrete systems being dealt with in the same way as, for instance, that obtained by replacing $x_{k+1} \rightarrow x_{k-1}$ in (2.31) with the initial condition $x_{0} \in A_{1}$ (and appropriate ad hoc re-definition of the mapping which generates the trajectory solution from given initial conditions) do not require such a consistency constraint. The dynamic system (2.31) is asymptotically linear if $\eta_{n}(x) \rightarrow 0$ as $n \rightarrow \infty ; \forall x \in \mathbf{R}^{p}$. Note that for the Euclidean distance (and norm), $d\left(x_{k+n}, x_{k+1}\right) \leq\left\|F_{n k}\right\| d\left(x_{k}, x_{k+1}\right)+\left\|\eta_{n k}\right\| ; \forall k \in \mathbf{N}$. Assume that the squared spectral norm of $F_{n k}$ is upper-bounded by $k_{n k}^{2}=k_{n}^{2}\left(x_{k}\right)=\frac{\alpha_{n k}+\beta_{n k}}{1-\beta_{n k}\left(1+2 \mu_{n k}\right)}$ for some parameterizing scalar sequences $\left\{\alpha_{n k}\right\},\left\{\beta_{n k}\right\}$ and $\left\{\mu_{n}\right\}$ which can be dependent, in a more general case, on the state $x_{k}$. This holds, for instance, if $F_{n k}=F_{n}\left(x_{k}\right)=\frac{1}{a_{n k}}\left(\frac{\alpha_{n k}+\beta_{n k}}{1-\beta_{n k}\left(1+2 \mu_{n k}\right)}\right)^{1 / 2} P_{n k}$, where $\left\{a_{n k}\right\}$ is a real positive sequence satisfying $a_{n k} \geq\left\|P_{n k}\right\|$ and $P_{n k}\left(=P_{n}\left(x_{k}\right)\right): \mathbf{N} \times \mathbf{R}^{p} \rightarrow \mathbf{R}^{p \times p}$ both being potentially dependent on the state as the rest of the parameterizing sequences. Since the spectral norm equalizes the spectral radius if the matrix is symmetric, then $k_{n}$ can be taken exactly as the spectral radius of $F_{n k}$ in such a case, i.e., it equalizes the absolute value of its dominant eigenvalue. We have to check the condition

$$
\limsup _{n \rightarrow \infty}\left(\left(1+2 \mu_{n k}-\beta_{n k}\right) d^{2}\left(T^{n} x_{k}, T^{n} y_{k}\right)-\left(\alpha_{n k}+\beta_{n k}\right) d^{2}\left(x_{k}, y_{k}\right)\right) \leq 0 ; \quad \forall k \in \mathbf{N}
$$

provided, for instance, that the distance is the Euclidean distance, induced by the Euclidean norm, then both being coincident, and provided also that we take the metric space $\left(\mathbf{R}^{p}, d\right)$ which holds, in particular, if

(a) $\left\{\left|\eta_{n k}\right|\right\} \subset \mathbf{R}_{0+},\left\{\alpha_{n k}\right\} \subset[1, \infty), \beta_{n k}=\beta \in[0,1),\left\{\mu_{n k}\right\} \subset\left[-1, \frac{1-\beta}{2 \beta}\right) ; \forall n, k \in \mathbf{N}, \alpha_{n k} \rightarrow 1$ and $\eta_{n k} \rightarrow 0, \mu_{n k} \rightarrow-1$, as $n \rightarrow \infty ; \forall k \in \mathbf{N}$. This implies that $k_{1 n k}^{2}=\frac{\alpha_{n k}+\beta}{1-\beta_{n k}\left(1+2 \mu_{n k}\right)} \geq 1$; $\forall n, k \in \mathbf{N}$ and $k_{1 n k} \rightarrow 1$ as $n \rightarrow \infty ; \forall k \in \mathbf{N}$. Thus, $T: \mathbf{R}^{p} \rightarrow \mathbf{R}^{p}$ is asymptotically nonexpansive being also an asymptotic strict $\beta$-pseudocontraction in the intermediate sense. This also implies that (2.31) is globally stable as it is proven as follows. Assume the contrary so that there is an infinite subsequence $L_{u}$ of $\left\{\left\|x_{n k}\right\|\right\}$ which is unbounded, and then there is also an infinite subsequence $L_{u a}$ which is strictly increasing. Since $\eta_{n k}=\eta_{n}\left(x_{k}\right) \rightarrow 0$ and $k_{1 n k}=k_{1 n}\left(x_{k}\right) \rightarrow 1$ as $n \rightarrow \infty ; \forall k \in \mathbf{N}$, one has that for $\forall x_{1} \in A_{1}$, any given $k \in \mathbf{N}$ and some 
sufficiently large $m_{01}=m_{01}\left(x_{k}\right), m_{02}=m_{02}\left(x_{k}\right) \in \mathbf{N}, \exists \varepsilon_{1}=\varepsilon_{1}\left(m_{01}\right) \in \mathbf{R}_{+}, \varepsilon_{2}=\varepsilon_{2}\left(m_{02}\right) \in \mathbf{R}_{+}$ such that $k_{m_{1}}=k_{m_{1}}\left(x_{k}\right) \leq 1+\varepsilon_{1}$ and $\left\|\eta_{m_{2}}\left(x_{k}\right)\right\| \leq \varepsilon_{2} ; \forall m_{1} \geq m_{01}, \forall m_{2} \geq m_{02}$. Now, take $m_{0}=\max \left(m_{01}, m_{02}\right)$ and $\varepsilon=\max \left(\varepsilon_{1}, \varepsilon_{2}\right)$. Then $\left\|x_{k+m}\right\| /\left\|x_{k}\right\| \leq 1+\varepsilon+\varepsilon /\left\|x_{k}\right\| ; \forall m(\in \mathbf{N}) \geq m_{0}$ and any given $k \in \mathbf{N}$. If $x_{k} \equiv 0$, then stability holds trivially. Assume not, and there are unbounded solutions. Thus, take $x_{k}(\neq 0), x_{k+m} \in L_{u a}$ such that $\left\|\frac{x_{k+m}}{x_{k}}\right\| \geq M$ for any given $M \in \mathbf{R}_{+}, \forall m(\in \mathbf{N}) \geq \bar{m}$ and some $\bar{m}=\bar{m}(M) \geq m_{0}$. Note that since $L_{u a}$ is a strictly increasing real sequence $\{M(\bar{m})\}$ implying $M(\bar{m}) \rightarrow \infty$ as $\bar{m} \rightarrow \infty$, which leads to a contradiction to the inequality $M \leq 1+\varepsilon\left(1+\frac{1}{\left\|x_{k}\right\|}\right)$ for $\varepsilon \rightarrow 0^{+}$for some sufficiently large $\bar{m}$, then for some sufficiently large $M$, if such a strictly increasing sequence $L_{u a}$ exists. Hence, there is no such sequence, and then no unbounded sequence $L_{u}$ for any initial condition in $A_{0}$. As a result, for any initial condition in any given subset $A_{1}$ of $\mathbf{R}^{p}$ (even if it is unbounded), any solution sequence of (2.31) is bounded, and then (2.31) is globally stable. The above reasoning implies that there is an infinite collection of numerable nonempty bounded closed sets $\left\{A_{i} \subset \mathbf{R}^{p}: i \in \mathbf{N}\right\}$, which are not necessarily connected, such that $x_{k} \in A_{k} ; \forall k \in \mathbf{N}$ and any given $x_{0} \in A_{0}$. Assume that the set $A_{0}$ of initial conditions is bounded, convex and closed and consider the collection of convex envelopes \{convex $A_{i} \subset \mathbf{R}^{p}: i \in \mathbf{N}$ \}, define constructively the closure convex set $C\left(A_{0}\right)=\operatorname{cl}\left(\operatorname{convex}\left(\bigcup_{i=1}^{\infty} \operatorname{convex} A_{i}\right)\right)$ which is trivially bounded, convex and closed. Note that it is not guaranteed that $\bigcup_{i=1}^{\infty}$ convex $A_{i}$ is either open or closed since there is a union of infinitely many closed sets involved. Note also that the convex hull of all the convex envelopes of the collection of sets is involved to ensure that $A$ is convex since the union of convex sets is not necessarily convex (so that $\bigcup_{i=1}^{\infty}$ convex $A_{i}$ is not guaranteed to be convex while $A$ is convex). Consider now the self-mapping $\bar{T}: C \rightarrow C$ which defines exactly the same solution as $T: \mathbf{R}^{p} \rightarrow \mathbf{R}^{p}$ for initial conditions in $A_{1}$ so that $\bar{T}$ is identified with the restricted self-mapping $T: \mathbf{R}^{p} \mid C \rightarrow C$ from a nonempty bounded, convex and closed set to itself. Note that $\left(\mathbf{R}^{p}, d\right)$ for the Euclidean distance is a convex metric space which is also complete since it is finite dimensional. Then $F_{n k}: A \subset \mathbf{R}^{p} \rightarrow \mathbf{R}^{p \times p}$ and $\eta_{n k}: A \subset \mathbf{R}^{p} \rightarrow \mathbf{R}^{p}$ are both continuous, then $\bar{T}: C \rightarrow C$ is also continuous and has a fixed point in $A$ from Theorem 2.8(i).

(b) If the self-mapping is asymptotically pseudocontractive in the intermediate sense, then the above conclusions still hold with the modification $k_{2 n k}^{2}=\frac{\alpha_{n k}+\beta_{n k}}{1-\beta_{n k}\left(1+2 \mu_{n k}\right)}(\geq 1) \rightarrow 1$ and $-\mu_{n k} \rightarrow \alpha_{n k} \rightarrow \beta_{n k} \rightarrow 1$ as $n \rightarrow \infty ; \forall k \in \mathbf{N}$. From Remark 2.7(2), $\beta_{n k}>\beta \Rightarrow k_{2 n k}>$ $k_{1 n k}$ and $\beta_{n k}<\beta \Rightarrow k_{2 n k}<k_{1 n k}$ for any $n, k \in \mathbf{N}$. Thus the convergence is guaranteed to be faster for an asymptotic $\beta$-strict pseudocontraction in the intermediate sense than for an asymptotic pseudocontraction in the intermediate sense with a sequence $\left\{\beta_{n k}\right\}$ such that $\beta_{n k}>\beta ; \forall n \in \mathbf{N}$ with the remaining parameters and parametrical sequences being identical in both cases. If $F_{n k}: A \subset \mathbf{R}^{p} \rightarrow \mathbf{R}^{p \times p}$ and $\eta_{n k}: A \subset \mathbf{R}^{p} \rightarrow \mathbf{R}^{p} ; \forall n, k \in \mathbf{N}$ are both continuous, then $\bar{T}: A \rightarrow A$ is continuous and has a fixed point in $A$ from Theorem 2.8(ii).

(c) If $T: X \rightarrow X$ is asymptotically $\beta$-strictly contractive in the intermediate sense, then $k_{3 n k}=\frac{\alpha_{n k}+\beta_{n k}}{1-\beta_{n k}\left(1+2 \mu_{n k}\right)} \rightarrow k_{3}=o(\max (\sqrt{\alpha}, \sqrt{\beta})) \in[0,1) ; \forall k \in \mathbf{N}$ so that it is asymptotically strictly contractive and has a unique fixed point from Theorem 2.8(iii).

(d) If $T: X \rightarrow X$ is asymptotically contractive in the intermediate sense, $k_{4 n k}=$ $\left(\frac{\alpha_{n k}+\beta_{n k}}{1-\beta_{n k}\left(1+2 \mu_{n k}\right)}\right)^{1 / 2} \rightarrow k_{4} \geq \frac{1}{\sqrt{2}}+o(\alpha) \geq \frac{1}{\sqrt{2}} ; \forall k \in \mathbf{N}$. Thus, $T: X \rightarrow X$ is an asymptotic strict contraction and has a unique fixed point from Theorem 2.8(iv).

Remark 2.10 Note that conditions like (2.32) can be tested on dynamic systems being different from (2.31) by redefining, in an appropriate way, the self-mapping which generates 
the solution sequence from given initial conditions. This allows to investigate the asymptotic properties of the self-mapping, the convergence of the solution to fixed points, then the system stability, etc. in a unified way for different dynamic systems. Close considerations can be discussed for different dynamic systems and convergence of the solutions generated by the different cyclic self-mappings defined on the union of several subsets to the best proximity points of each of the involved subsets.

\section{Asymptotic contractions and pseudocontractions of cyclic self-mappings in the intermediate sense}

Let $A, B \subset X$ be nonempty subsets of $X . T: A \cup B \rightarrow A \cup B$ is a cyclic self-mapping if $T(A) \subseteq B$ and $T(B) \subseteq A$. Assume that the asymptotically nonexpansive condition (2.10), subject to (2.11), is modified as follows:

$$
\begin{aligned}
& d^{2}\left(T^{n} x, T^{n} y\right) \leq \alpha_{n}(x, y) d^{2}(x, y)+\beta_{n}(x, y)\left(d^{2}(x, y)+d^{2}\left(T^{n} x, T^{n} y\right)\right) \\
& +2 \mu_{n}(x, y) \beta_{n}(x, y) d(x, y) d\left(T^{n} x, T^{n} y\right) \\
& +\xi_{n}(x, y)+\gamma_{n}(x, y) D^{2} ; \quad \forall x \in A, y \in B, \forall n \in \mathbf{N}, \\
& \xi_{n}=\xi_{n}(x, y) \\
& :=\max \left(0,\left(1-\beta_{n}(x, y)\right) d^{2}\left(T^{n} x, T^{n} y\right)-\left(\alpha_{n}(x, y)+\beta_{n}(x, y)\right) d^{2}(x, y)\right. \\
& \left.-2 \mu_{n}(x, y) \beta_{n}(x, y) d(x, y) d\left(T^{n} x, T^{n} y\right)\right) ; \quad \forall x \in A, y \in B, \forall n \in \mathbf{N}
\end{aligned}
$$

with $\left(\xi_{n}-\gamma_{n}(x, y) D^{2}\right) \rightarrow 0 ; \forall x, y \in X$ as $n \rightarrow \infty$, and that the asymptotically nonexpansive condition (2.22), subject to (2.23), is modified as follows:

$$
\begin{aligned}
\begin{aligned}
d^{2}\left(T^{n} x, T^{n} y\right) \leq & \alpha_{n}(x, y) d^{2}(x, y)+\beta_{n}(x, y)\left(d^{2}(x, y)+d^{2}\left(T^{n} x, T^{n} y\right)\right) \\
& +2 \mu_{n}(x, y) \beta_{n}(x, y) d^{2}\left(T^{n} x, T^{n} y\right) \\
& +\xi_{n}(x, y)+\gamma_{n}(x, y) D^{2} ; \quad \forall x \in A, y \in B, \forall n \in \mathbf{N},
\end{aligned} \\
\begin{aligned}
& \xi_{n}= \xi_{n}(x, y) \quad \\
&:=\max \left(0,\left(1-\beta_{n}(x, y)\right) d^{2}\left(T^{n} x, T^{n} y\right)-\left(\alpha_{n}(x, y)+\beta_{n}(x, y)\right) d^{2}(x, y)\right.
\end{aligned} \\
\left.-2 \mu_{n}(x, y) \beta_{n}(x, y) d\left(T^{n} x, T^{n} y\right)\right) ; \quad \forall x, y \in X \text { as } n \rightarrow \infty
\end{aligned}
$$

with $\left(\xi_{n}-\gamma_{n}(x, y) D^{2}\right) \rightarrow 0 ; \forall x, y \in X$ as $n \rightarrow \infty$, where $\left\{\gamma_{n}(x, y)\right\} \in[0, \infty)$ and $D=$ $\operatorname{dist}(A, B) \geq 0$. If $A \cap B \neq \varnothing$, then $D=0$ and Theorems 2.1, 2.2 and 2.8 hold with the replacement $A \rightarrow A \cap B$. Then if $A$ and $B$ are closed and convex, then there is a unique fixed point of $T: A \cup B \rightarrow A \cup B$ in $A \cap B$. In the following, we consider the case that $A \cap B=\varnothing$ so that $D>0$. The subsequent result based on Theorems 2.1, 2.2 and 2.8 holds.

Theorem 3.1 Let $(X, d)$ be a metric space and let $T: A \cup B \rightarrow A \cup B$ be a cyclic selfmapping, i.e., $T(A) \subseteq B$ and $T(B) \subseteq A$, where $A$ and $B$ are nonempty subsets of $X$. Define the sequence $\left\{k_{n}\right\}_{n \in \mathbf{N}} \subset[0, \infty)$ of asymptotically nonexpansive iteration-dependent constants 
as follows:

$$
k_{n}=k_{n}(x, y):= \begin{cases}\frac{\alpha_{n}+\beta_{n}\left(1+2 \mu_{n}\right)}{1-\beta_{n}} \leq 1 & \text { if } d\left(T^{n} x, T^{n} y\right) \leq d(x, y) ; \\ \frac{\alpha_{n}+\beta_{n}}{1-\beta_{n}\left(1+2 \mu_{n}\right)} \geq 1 & \text { if } d\left(T^{n} x, T^{n} y\right) \geq d(x, y) ;\end{cases}
$$

$\forall(x, y) \in(A \times B) \cup(B \times A), \forall n \in \mathbf{N}$ provided that $T: A \cup B \rightarrow A \cup B$ satisfies the constraint (3.1), subject to (3.2), and

$$
\begin{aligned}
& \quad\left[\left(d\left(T^{n} x, T^{n} y\right) \leq d(x, y) \wedge \beta_{n}=1\right)\right. \\
& \left.\quad \Rightarrow\left(\gamma_{n}=0\right), \forall(x, y) \in(A \times B) \cup(B \times A), \forall n \in \mathbf{N}\right]
\end{aligned}
$$

and

$$
k_{n}=k_{n}(x, y)=\frac{\alpha_{n}+\beta_{n}}{1-\beta_{n}\left(1+2 \mu_{n}\right)} \geq 1 ;
$$

$\forall n \in \mathbf{N}$ for $x \in A(y \in B)$ and for $x \in B(y \in A)$ provided that $T: A \cup B \rightarrow A \cup B$ satisfies the constraint (3.3) subject to (3.4) provided that the parameterizing bounded real sequences $\left\{\alpha_{n}(x, y)\right\},\left\{\beta_{n}(x, y)\right\},\left\{\mu_{n}(x, y)\right\}$ and $\left\{\gamma_{n}(x, y)\right\}$ of general terms $\alpha_{n}=\alpha_{n}(x, y), \beta_{n}=\beta_{n}(x, y)$ and $\mu_{n}=\mu_{n}(x, y)$ fulfill the following constraints:

$$
\begin{aligned}
& {\left[\left(\mu_{n}(x, y) \in\left[-\frac{\alpha_{n}(x, y)+\beta_{n}(x, y)}{2 \beta_{n}(x, y)}, \frac{1-\alpha_{n}(x, y)-2 \beta_{n}(x, y)}{2 \beta_{n}(x, y) d(x, y)}\right]\right) \wedge\left(\beta_{n}(x, y)<1\right)\right]} \\
& \quad \vee\left[\left(\mu_{n}(x, y)<-\frac{\alpha_{n}(x, y)+\beta_{n}(x, y)}{2 \beta_{n}(x, y)}\right) \wedge\left(\beta_{n}(x, y)>1\right) \Leftrightarrow \xi_{n}(x, y)=0\right] \\
& \quad \vee\left[\mu_{n}(x, y) \in\left[\frac{1-\alpha_{n}(x, y)-2 \beta_{n}(x, y)}{2 \beta_{n}(x, y)}, \frac{1-\beta_{n}(x, y)}{2 \beta_{n}(x, y)}\right)\right] ; \\
& \quad \forall(x, y) \in(A \times B) \cup(B \times A), \forall n \in \mathbf{N},
\end{aligned}
$$

$\gamma_{n}=\gamma_{n}(x, y) \geq \max \left(0,1-k_{n}\right)$ and assuming that the following limits exist:

$$
\begin{aligned}
& \left(\mu_{n}(x, y)-\frac{1-\alpha_{n}(x, y)-2 \beta_{n}(x, y)}{2 \beta_{n}(x, y)}\right) \rightarrow 0 \\
& \Leftrightarrow \quad \alpha_{n}+2 \beta_{n}\left(1+\mu_{n}\right) \rightarrow 1 ; \quad \gamma_{n}(x, y) \rightarrow 0 ; \\
& \forall(x, y) \in(A \times B) \cup(B \times A) \text { as } n \rightarrow \infty .
\end{aligned}
$$

Then, the following properties hold:

(i) $T: A \cup B \rightarrow A \cup B$ satisfies (3.3) subject to (3.4)-(3.9); $\forall(x, y) \in(A \times B) \cup(B \times A)$. Then

$$
\exists \lim _{n \rightarrow \infty} d\left(T^{n} x, T^{n} y\right) \in[D, d(x, y)] ; \quad \forall(x, y) \in(A \times B) \cup(B \times A)
$$

so that $T: A \cup B \rightarrow A \cup B$ is a cyclic asymptotically nonexpansive self-mapping. If $x \in A$ is a best proximity point of $A$ and $y \in B$ is a best proximity point of $B$, then $\lim _{n \rightarrow \infty} d\left(T^{n} x, T^{n} y\right)=D$ and $T^{2 n} x \rightarrow z_{x}=z(x)$ and $T^{2 n} y \rightarrow z_{y}=z(y)$, which are best 
proximity points of $A$ and $B$ (not being necessarily identical to $x$ and $y$ ), respectively if $T: A \cup B \rightarrow A \cup B$ is continuous.

(ii) Property (i) also holds if $T: A \cup B \rightarrow A \cup B$ satisfies (3.1) subject to (3.2), (3.7), (3.8)(3.9) and (3.5b) provided that $d\left(T^{n} x, T^{n} y\right) \geq d(x, y) ; \forall(x, y) \in(A \times B) \cup(B \times A)$.

Proof The second condition of (2.18) now becomes under either (3.1)-(3.2) and (3.8)-(3.9)

$$
\begin{aligned}
& d\left(T^{n} x, T^{n} y\right)^{2} \leq k_{b n} d^{2}(x, y)+\frac{\xi_{n}+\gamma_{n} D^{2}}{1-\beta_{n}} \\
& \quad \Rightarrow \quad \exists \lim _{n \rightarrow \infty} d\left(T^{n} x, T^{n} y\right) \in[D, d(x, y)] ; \quad \forall(x, y) \in(A \times B) \cup(B \times A),
\end{aligned}
$$

and it now becomes under (3.3)-(3.4) and (3.8)-(3.9)

$$
\begin{aligned}
& d\left(T^{n} x, T^{n} y\right)^{2} \leq k_{b n} d^{2}(x, y)+\frac{\xi_{n}+\gamma_{n} D^{2}}{1-\beta_{n}\left(1+2 \mu_{n}\right)} \\
& \quad \Rightarrow \quad \exists \lim _{n \rightarrow \infty} d\left(T^{n} x, T^{n} y\right) \in[D, d(x, y)] ; \quad \forall(x, y) \in(A \times B) \cup(B \times A)
\end{aligned}
$$

since $T^{n} x, T^{n} x \in A \cup B ; \forall n \in \mathbf{N}$ since $T(A) \subseteq B$ and $T(B) \subseteq A$, and $k_{n} \rightarrow 1$ and $\gamma_{n}(x, y) \rightarrow$ $\left(1-k_{n}\right) \rightarrow 0$ as $n \rightarrow \infty ; \forall(x, y) \in(A \times B) \cup(B \times A)$. Note that (3.8) implies that there is no division by zero in (3.11). Now, assume that (3.10) holds with $\beta_{n}=1$. From (3.8) and (3.2), $\mu_{n} \in\left[-\frac{1+\alpha_{n}}{2}, 0\right]$, equivalently, $\left|\mu_{n}\right| \leq \frac{1+\alpha_{n}}{2}$ and $d\left(T^{n} x, T^{n} y\right)>\frac{\alpha_{n}+1}{2\left|\mu_{n}\right|} d^{2}(x, y) \geq d^{2}(x, y)$, which contradicts (3.5a) if $\xi_{n}>0$ so that $\beta_{n}=1$ in (3.5a) under (3.7) implies that $\xi_{n}=0$ and, since $\gamma_{n}=0$ from (3.6), there is no division by zero on the right-hand side of (3.10) if $\beta_{n}=1$.

Also, if $T: A \cup B \rightarrow A \cup B$ is continuous, then $\lim _{n \rightarrow \infty} d\left(T^{2 n} x, T^{2 n} y\right)=d\left(\lim _{n \rightarrow \infty} T^{2 n} x\right.$, $\left.\lim _{n \rightarrow \infty} T^{2 n} y\right)=D$ so that $T^{2 n} x \in A ; \forall n \in \mathbf{N}, \lim _{n \rightarrow \infty} T^{2 n} x \in \operatorname{cl} A, T^{2 n} y \in B$ and $\lim _{n \rightarrow \infty} T^{2 n} x \in \operatorname{cl} B$ since $T(A) \subseteq B$ and $T(B) \subseteq A$. This proves Properties (i)-(ii).

Remark 3.2 Note that Theorem 3.1 does not guarantee the convergence of $\left\{T^{2 n} x\right\}$ and $\left\{T^{2 n} y\right\}$ to best proximity points if the initial points for the iterations $x \in A$ and $y \in B$ are not best proximity points if $T: A \cup B \rightarrow A \cup B$ is not contractive.

The following result specifies Theorem 3.1 for asymptotically nonexpansive mappings with $k_{n}=\frac{\alpha_{n}+\beta_{n}\left(1+2 \mu_{n}\right)}{1-\beta_{n}}<1 ; \forall n \in \mathbf{N}$ subject to $\lim _{n \rightarrow \infty} k_{n}=k_{c} \leq 1$.

Theorem 3.3 Let $(X, d)$ be a metric space and let $T: A \cup B \rightarrow A \cup B$ be a cyclic selfmapping which satisfies the asymptotically nonexpansive constraint (3.1), subject to (3.2), where $A$ and $B$ are nonempty subsets of $X$. Let the sequence $\left\{k_{n}\right\}_{n \in \mathbf{N}} \subset[0,1)$ of asymptotically nonexpansive iteration-dependent constants be defined by a general term $k_{n}(x, y)=$ $k_{n}:=\frac{\alpha_{n}+\beta_{n}\left(1+2 \mu_{n}\right)}{1-\beta_{n}} \in[0,1)$ under the constraints $\gamma_{n}(x, y)=\gamma_{n}:=\delta_{j}\left(1-k_{n}\right)\left(1-\beta_{n}\right)=o\left(1-\beta_{n}\right)$, $\beta_{n} \leq 1 \Rightarrow \mu_{n} \leq-\frac{1+\alpha_{n}}{2}, \forall n \in \mathbf{N}$ and $\lim _{n \rightarrow \infty} k_{n}=1$. Then the subsequent properties hold:

(i) The following limits exist:

$$
\begin{aligned}
& \lim _{n \rightarrow \infty} d\left(T^{n} x, T^{n} y\right)=D ; \quad \forall(x, y) \in(A \times B) \cup(B \times A) ; \\
& \lim _{n \rightarrow \infty} d\left(T^{n} x, T^{n+1} x\right)=D ; \quad \forall x \in A \cup B .
\end{aligned}
$$

(ii) Assume, furthermore, that $(X, d)$ is complete, $A$ and $B$ are closed and convex and $d$ : $X \times X$ is translation-invariant and homogeneous and $(X, d) \equiv(X,\|\|)$ is uniformly convex 
where $\|$ || is the metric-induced norm. Then

$$
\lim _{n \rightarrow \infty} d\left(T^{2 n} x, T^{2 n+2} x\right)=\lim _{n \rightarrow \infty} d\left(T^{2 n+1} x, T^{2 n+3} x\right)=0 ; \quad \forall x \in A \cup B,
$$

$\left\{T^{2 n} x\right\} \rightarrow z \in A,\left\{T^{2 n+1} x\right\} \rightarrow T z \in B ; \forall x \in A$, and $\left\{T^{2 n} y\right\} \rightarrow T z \in B,\left\{T^{2 n+1} x\right\} \rightarrow z \in A$; $\forall x \in A, \forall y \in B$, where $z$ and $T z$ are unique best proximity points in $A$ and $B$, respectively. If $A \cap B \neq \varnothing$, then $z=$ Tz is the unique fixed point of $T: A \cup B \rightarrow A \cup B$.

Proof Note from (3.9), under (3.6) and (3.7), that there is no division by zero on the righthand side of (3.10) and $\xi_{n}=\gamma_{n}=0$ if $\beta_{n}=1$. Then one has from (3.1)-(3.2), (3.5a), (3.6) and (3.7) that

$$
d^{2}\left(T^{(j+1) n} x, T^{(j+1) n} y\right) \leq k_{j n} d^{2}\left(T^{j n} x, T^{j n} y\right)+\left(1-k_{j n}\right) D^{2}+\frac{\xi_{j n}}{1-\beta_{j n}} ; \quad \forall j, n \in \mathbf{N} .
$$

There are several possible cases as follows.

Case A: $\left\{d\left(T^{j n} x, T^{j n} y\right)\right\}$ is non-increasing. Then $d\left(T^{j n} x, T^{j n} y\right) \rightarrow g=g(x, y) \geq D$ as $n \rightarrow \infty ; \forall(x, y) \in(A \times B) \cup(B \times A)$. Since $\left\{k_{n}\right\} \subset[0,1)$, one gets (3.12).

Case B: $\left\{d\left(T^{j n} x, T^{j n} y\right)\right\}$ is non-decreasing. Then either $\left\{d\left(T^{j n} x, T^{j n} y\right)\right\} d\left(T^{j n} x, T^{j n} y\right) \rightarrow g=$ $g(x, y) \geq D$ as $n \rightarrow \infty ; \forall(x, y) \in(A \times B) \cup(B \times A)$ or it is unbounded. Then it has a subsequence which diverges, from which a strictly increasing subsequence can be taken. But this contradicts $\lim \sup _{n \rightarrow \infty}\left(d^{2}\left(T^{(j+1) n} x, T^{(j+1) n} y\right)-d^{2}\left(T^{j n} x, T^{j n} y\right)\right) \leq 0$ following from (3.14) subject to the given parametrical constraints. Thus, if $\left\{d\left(T^{j n} x, T^{j n} y\right)\right\}$ is non-decreasing, it cannot have a strictly increasing subsequence so that it is bounded and has a finite limit as in Case A.

Case C: $\left\{d\left(T^{j n} x, T^{j n} y\right)\right\}$ has an oscillating subsequence. It is proven that such a subsequence is finite. Assume not, then if $\lim _{\sup _{n \rightarrow \infty}}\left(d^{2}\left(T^{(j+1) n} x, T^{(j+1) n} y\right)-d^{2}\left(T^{j n} x, T^{j n} y\right)\right) \leq 0$, there is an integer sequence $\left\{p_{n}\right\}$ of general term subject to $p_{n} \in(n, 2 n)$ such that

$$
\limsup _{n \rightarrow \infty}\left(d^{2}\left(T^{(j+1) n+p_{n}} x, T^{(j+1) n+p_{n}} y\right)-d^{2}\left(T^{j n+p_{n}} x, T^{j n+p_{n}} y\right)\right)>0
$$

but the above expression is equivalent, for $x_{p n}=T^{p_{n}} x$ and $y_{p n}=T^{p_{n}} y$ which are in $A \cup B$, but not jointly in either $A$ or $B$, to

$$
\limsup _{n \rightarrow \infty}\left(d^{2}\left(T^{(j+1) n} x_{p n}, T^{(j+1) n} y_{p n}\right)-d^{2}\left(T^{j n+p_{n}} x_{p n}, T^{j n+p_{n}} y_{p n}\right)\right)>0,
$$

which contradicts $\limsup _{n \rightarrow \infty}\left(d^{2}\left(T^{(j+1) n} x, T^{(j+1) n} y\right)-d^{2}\left(T^{j n} x, T^{j n} y\right)\right) \leq 0$ since both sequences $\left\{T^{j n} x\right\}$ and $\left\{T^{j n} y\right\}$ are bounded; $\forall(x, y) \in(A \times B) \cup(B \times A)$. Then there is no infinite oscillating sequence $\left\{d\left(T^{j n} x, T^{j n} y\right)\right\}$ for some $(x, y) \in(A \times B) \cup(B \times A)$ so that there is a finite limit $g=g(x, y) \geq D$ of $\left\{d\left(T^{j n} x, T^{j n} y\right)\right\}, \forall(x, y) \in(A \times B) \cup(B \times A)$. Now, proceed by contradiction by assuming the existence of some $(x, y) \in(A \times B) \cup(B \times A)$ such that $d\left(T^{j n} x, T^{j n} y\right) \rightarrow g=g(x, y)=D+\varepsilon>0$ as $n \rightarrow \infty ; \forall j \in \mathbf{N}$. Thus, for any $j, n_{0} \in \mathbf{N}$, there is some $n\left(\geq n_{0}\right) \in \mathbf{N}$ such that there are two consecutive nonzero elements of a nonzero real sequence $\left\{\varepsilon_{n}\right\}$, which can depend on $x$ and $y$, which satisfy $\varepsilon_{n+1} \geq \varepsilon_{n}$ and

$$
d\left(T^{j n} x, T^{j n} y\right)=D+\varepsilon_{n+1} \geq d\left(T^{j n} x, T^{j n} y\right)=D+\varepsilon_{n} ;
$$


$\forall j \in \mathbf{N}$. Otherwise, if $\varepsilon_{n+1}<\varepsilon_{n}$ for any $n(\in \mathbf{N}) \geq n_{0}$ and any given $j, n_{0} \in \mathbf{N}$ and $(x, y) \in$ $(A \times B) \cup(B \times A)$, then $d\left(T^{j n} x, T^{j n} y\right) \rightarrow D$ as $n \rightarrow \infty ; \forall(x, y) \in(A \times B) \cup(B \times A)$. One gets, by combining (3.14) and (3.15), that

$$
\begin{aligned}
&\left(D+\varepsilon_{n}\right)^{2} \leq\left(D+\varepsilon_{n+1}\right)^{2} \\
&=d^{2}\left(T^{(j+1) n} x, T^{(j+1) n} y\right) \\
& \leq k_{j n} d^{2}\left(T^{j n} x, T^{j n} y\right)+\left(1-k_{j n}\right) D^{2}+\frac{\xi_{j n}}{1-\beta_{j n}} \\
&=k_{j n}\left(D+\varepsilon_{n}\right)^{2}+\left(1-k_{j n}\right) D^{2}+\frac{\xi_{j n}}{1-\beta_{j n}} \\
&=k_{j n}\left(D+\varepsilon_{n}\right)^{2}+\left(1+\delta_{j n}+\rho_{j n}\right)\left(1-k_{j n}\right) D^{2} \\
& \Rightarrow \quad\left(D+\varepsilon_{n}\right)^{2} \leq\left(1+\delta_{j n}+\rho_{j n}\right) D^{2} ;
\end{aligned}
$$

$\forall j, n \in \mathbf{N}$ since $k_{j n}<1 ; \forall j, n \in \mathbf{N}$, and some nonnegative real sequence $\left\{\rho_{j n}\right\}$ which converges to zero since $\xi_{j n} \rightarrow \gamma_{j n} D^{2} \rightarrow \delta_{j}\left(1-k_{j n}\right)\left(1-\beta_{j n}\right) D^{2}=o\left(1-\beta_{j n}\right) \rightarrow 0$ as $n \rightarrow \infty ; \forall j \in \mathbf{N}$ for any $(x, y) \in(A \times B) \cup(B \times A)$ so that $\frac{\xi_{j n}}{1-\beta_{j n}}=\frac{1-k_{j n}}{\gamma_{j n}} \xi_{j n} \delta_{j n}(\forall n \in \mathbf{N}) \rightarrow\left(1-k_{j n}\right) \delta_{j n} D^{2}+\rho_{j n}$ as $n \rightarrow \infty$; $\forall j \in \mathbf{N}$. The relations (3.16) contradict $\limsup _{n \rightarrow \infty}\left\lfloor\left(D+\varepsilon_{n}\right)^{2}-\left(1+\delta_{j n}+\rho_{j n}\right) D^{2}\right\rfloor>0$ since $\left\{\varepsilon_{n}\right\}$ is positive $\forall n \in \mathbf{N}$ (and it does not converge to zero) and $\rho_{j n} \rightarrow 0, \delta_{j n} \rightarrow 0$ as $n \rightarrow \infty$. Thus, one concludes that $\left\{\varepsilon_{n}\right\}$ converges to zero, and then $\lim _{n \rightarrow \infty} d\left(T^{n} x, T^{n} y\right)=D$; $\forall(x, y) \in(A \times B) \cup(B \times A) ; \forall(x, y) \in(A \times B) \cup(B \times A)$. This leads to $\lim _{n \rightarrow \infty} d\left(T^{n} x, T^{n+1} x\right)=$ $D ; \forall x \in A \cup B$ by taking $y=T x$ with $y \in B$ if $x \in A$ and $y \in A$ if $x \in B$. Property (i) has been proven.

Now, Property (ii) is proven. It is first proven that $\lim _{n \rightarrow \infty} d\left(T^{2 n} x, T^{2 n+2} x\right)=$ $\lim _{n \rightarrow \infty} d\left(T^{2 n+1} x, T^{2 n+3} x\right)=0 ; \forall x \in A \cup B$ if the metric is translation-invariant and homogeneous so that it induces a norm \|\| if $A$ and $B$ are nonempty, closed and convex subsets of $X$ and $(X,\|\|) \equiv(X, d)$ is a uniformly convex Banach space. Assume not and take such a norm to yield $d\left(T^{2 n} x, T^{2 n+2} x\right)>0$. Then if $A$ is nonempty, closed and convex and $B$ is nonempty and closed and $x \in A$, then $\left\{T^{2 n} x\right\},\left\{T^{2 n+2} x\right\} \subset A$. It is known that $d\left(T^{2 n} x, T^{2 n+2} x\right) \rightarrow d_{x} \leq d\left(x, T^{2} x\right)$ from Theorem 3.1(i) for $y=T^{2} x$. Since $(X,\|\|) \equiv(X, d)$ is a uniformly convex Banach space for the metric-induced norm (being equivalent to the translation-invariant homogeneous metric), we have the following property for the sequences $\left\{T^{2 n} x\right\},\left\{T^{2 n+2} x\right\} \subset A$ and $\left\{p_{n}\right\} \subset X$ satisfying for some strictly increasing nonnegative sequence of functions $\left\{\delta_{n}:\left[0, \frac{r_{2 n}}{R_{2 n}}\right] \rightarrow \mathbf{R}_{0+}\right\}$ and any nonnegative sequences $\left\{r_{2 n}\right\}$ and $\left\{R_{2 n}\right\}$ satisfying $r_{2 n} \leq R_{2 n}$ and any sequence $\left\{p_{2 n}\right\} \subset X ; \forall n \in \mathbf{N}$ that

$$
\begin{gathered}
\max \left(d\left(T^{2 n} x, p_{2 n}\right), d\left(T^{2 n+2} x, p_{2 n}\right)\right)=\max \left(\left\|T^{2 n} x-p_{2 n}\right\|,\left\|T^{2 n+2} x-p_{2 n}\right\|\right) \\
\leq R_{2 n}, \\
r_{2 n} \leq d\left(T^{2 n} x, T^{2 n+2} x\right)=\left\|T^{2 n+2} x-T^{2 n} x\right\| \\
\leq\left\|T^{2 n+2} x\right\|+\left\|T^{2 n} x\right\|, \\
\left|\left\|T^{2 n} x+T^{2 n+2} x\right\|-2\left\|p_{n}\right\|\right| \leq\left\|T^{2 n} x+T^{2 n+2} x-2 p_{n 2}\right\| \\
\leq 2\left(1-\delta\left(\frac{r_{2 n}}{R_{2 n}}\right)\right) R_{2 n} ;
\end{gathered}
$$


$\forall x \in A \cup B, \forall n \in \mathbf{N}$, which implies that

$$
\begin{aligned}
\max \left(r_{2 n}, 2\left[\left\|p_{2 n}\right\|-\left(1-\delta\left(\frac{r_{2 n}}{R_{2 n}}\right)\right) R_{2 n}\right]\right) & \leq\left\|T^{2 n} x+T^{2 n+2} x\right\| \\
& \leq 2\left[\left(1-\delta\left(\frac{r_{2 n}}{R_{2 n}}\right)\right) R_{2 n}+\left\|p_{2 n}\right\|\right]
\end{aligned}
$$

which has to be valid for $R_{2 n}=\frac{\left\|p_{2 n}\right\|}{1-\delta(2)} \leq(1-\delta(2)) \frac{\left\|p_{2 n}\right\|}{1-\delta(2)}+\left\|p_{2 n}\right\|=2\left\|p_{2 n}\right\| ; \forall n \in \mathbf{N}$. Now, for $p_{2 n}(\in X) \neq 0$ and $R_{2 n}=\left\|p_{2 n}\right\|>0 ; \forall n \in \mathbf{N}$, it follows that $\delta(2)=\delta(s)=0 ; \forall s \in[0,2]$, which is a contradiction to $\delta_{n}:[0,2] \rightarrow \mathbf{R}_{0+}$ being strictly increasing, then contradicting $(X,\|\|)$ being a uniformly convex Banach space, unless $r_{2 n} \rightarrow 2 R_{2 n} \rightarrow 0$ as $n \rightarrow \infty$ so that $\left\{\delta_{n}:\left[0, \frac{r_{2 n}}{R_{2 n}}\right] \rightarrow \mathbf{R}_{0+}\right\}$ converges to $\delta(0)=0$. Taking $x \in A, p_{2 n}=T^{2 n+2} x \in A ; \forall n \in \mathbf{N}$, (3.15) for $r_{2 n} \rightarrow R_{2 n} \rightarrow 0$ as $n \rightarrow \infty$ implies the existence of the first zero limit in (3.13). The existence of the second zero limit in (3.13) is proven in the same way since $T x \in B$. Since those limits are zero, $\left\{T^{2 n} x\right\},\left\{T^{2 n+2} x\right\}$ are Cauchy sequences in $A$ converging to a best proximity point $z \in A$ for $x \in A$. Note that $z \in A$ is necessarily the unique best proximity point in $A$ since $\left\{T^{2 n} x\right\}$ and $\left\{T^{2 n}\left(T^{2} x\right)\right\}$ converge to the same point. Otherwise, the first limit of (3.13) would not exist if the sequences do not converge, then a contradiction holds to a proven result, and also Property (i) would not be true, since (3.12) would not hold, if the limit of the sequence would not be a best proximity point in $A$, then a contradiction holds to another proven result. In the same way, $\left\{T^{2 n+1} x\right\},\left\{T^{2 n+3} x\right\}$ converge to a unique best proximity point $z_{1} \in B$ for any $x \in A$. Now, $z_{1}=T z$. Assume not. Then since $\left\{T^{2 n} x\right\} \rightarrow z$, $\left\{T^{2 n+1} x\right\} \rightarrow z_{1}$ and $d\left(T^{2 n} x, T^{2 n+1} x\right) \rightarrow D$, one has $d\left(z, z_{1}\right)=D$. Assume that $z_{1} \neq T z$ so that since $A$ and $B$ are convex,

$$
D=d\left(z, z_{1}\right)=2\left\|\frac{z}{2}-\frac{z_{1}}{2}\right\|=2\left\|\left(\frac{z}{2}-\frac{T z}{2}\right)+\left(\frac{T z}{2}-\frac{z_{1}}{2}\right)\right\|>D
$$

which is a contradiction. Then $z_{1}=T z$ is the unique best proximity of $B$. If $A \cap B=\varnothing$, then $z_{1}=z=T z$ is the unique fixed point of $T: A \cup B \rightarrow A \cup B$ which coincides with the unique best proximity point in $A$ and $B$.

Remark 3.4 Theorem 3.3 is known for strictly contractive cyclic self-mappings [20] satisfying the contractive condition (3.1) in the case that $\xi_{n} \equiv 0$ and $k_{n}=\alpha_{n}<1, \beta_{n}=\xi_{n}=0$ and $\gamma_{n}=1-\alpha_{n}[5-7]$.

It is now assumed that the cyclic self-mapping $T: A \cup B \rightarrow A \cup B$ is asymptotically nonexpansive while not being strictly contractive for any finite number of iterations. The concepts of cyclic pseudocontractions and a strict contraction in the intermediate sense play an important role in the obtained results.

Theorem 3.5 Let $(X,\|\|)$ be a uniformly convex Banach space endowed with a metricinduced norm \| \| from a translation-invariant homogeneous metric $d: X \times X \rightarrow \mathbf{R}_{0+}$, where $A$ and $B \subset X$ are nonempty, closed and convex subsets of $X$ and assume that $T: A \cup$ $B \rightarrow A \cup B$ is a cyclic self-mapping. Define the sequence $\left\{k_{n}\right\}_{n \in \mathbf{N}} \subset[0, \infty)$ of asymptotically 
nonexpansive iteration-dependent constants as follows:

$$
k_{n}=k_{n}(x, y):= \begin{cases}\frac{\alpha_{n}+\beta_{n}\left(1+2 \mu_{n}\right)}{1-\beta_{n}} \leq 1 & \text { if } d\left(T^{n} x, T^{n} y\right)<d(x, y) ; \\ \frac{\alpha_{n}+\beta_{n}}{1-\beta_{n}\left(1+2 \mu_{n}\right)} \geq 1 & \text { if } d\left(T^{n} x, T^{n} y\right) \geq d(x, y) ;\end{cases}
$$

$\forall(x, y) \in(A \times B) \cup(B \times A), \forall n \in \mathbf{N}$ provided that $T: A \cup B \rightarrow A \cup B$ satisfies the constraint (3.1), subject to (3.2); and

$$
k_{n}=k_{n}(x, y)=\frac{\alpha_{n}+\beta_{n}}{1-\beta_{n}\left(1+2 \mu_{n}\right)} \geq 1
$$

$\forall n \in \mathbf{N}$ for $x \in A(y \in B)$ and for $x \in B(y \in A)$ provided that $T: A \cup B \rightarrow A \cup B$ satisfies the constraint (3.3), subject to (3.4), provided that the parameterizing bounded real sequences $\left\{\alpha_{n}(x, y)\right\},\left\{\beta_{n}(x, y)\right\},\left\{\mu_{n}(x, y)\right\}$ and $\left\{\gamma_{n}(x, y)\right\}$ of general terms $\alpha_{n}=\alpha_{n}(x, y), \beta_{n}=\beta_{n}(x, y)$ and $\mu_{n}=\mu_{n}(x, y)$ fulfill the following constraints:

$$
\begin{aligned}
& {\left[\left(\mu_{n}(x, y) \in\left[-\frac{\alpha_{n}(x, y)+\beta_{n}(x, y)}{2 \beta_{n}(x, y)}, \frac{1-\alpha_{n}(x, y)-2 \beta_{n}(x, y)}{2 \beta_{n}(x, y) d(x, y)}\right]\right) \wedge\left(\beta_{n}(x, y)<1\right)\right]} \\
& \quad \vee\left[\left(\mu_{n}(x, y)<-\frac{\alpha_{n}(x, y)+\beta_{n}(x, y)}{2 \beta_{n}(x, y)}\right) \wedge\left(\beta_{n}(x, y)>1\right) \Leftrightarrow \xi_{n}(x, y)=0\right] \\
& \quad \vee\left[\mu_{n}(x, y) \in\left[\frac{1-\alpha_{n}(x, y)-2 \beta_{n}(x, y)}{2 \beta_{n}(x, y)}, \frac{1-\beta_{n}(x, y)}{2 \beta_{n}(x, y)}\right)\right] ; \\
& \quad \forall(x, y) \in(A \times B) \cup(B \times A), \forall n \in \mathbf{N},
\end{aligned}
$$

$\gamma_{n}=\gamma_{n}(x, y) \geq \max \left(0,1-k_{n}\right)$ and assuming that the following limits exist:

$$
\begin{aligned}
\mu_{n}(x, y) & \rightarrow \frac{1-\alpha_{n}(x, y)-2 \beta_{n}(x, y)}{2 \beta_{n}(x, y)} \Leftrightarrow \alpha_{n}+2 \beta_{n}\left(1+\mu_{n}\right) \rightarrow 1 ; \quad \gamma_{n}(x, y) \rightarrow 0 ; \\
\forall(x, y) & \in(A \cup B) \times(B \cup A) \text { as } n \rightarrow \infty .
\end{aligned}
$$

Then the following properties hold:

(i) If $T: A \cup B \rightarrow A \cup B$ satisfies (3.3) subject to (3.20)-(3.24); $\forall(x, y) \in(A \times B) \cup(B \times A)$, then

$$
\exists \lim _{n \rightarrow \infty} d\left(T^{n} x, T^{n} y\right) \in[D, d(x, y)] ; \quad \forall(x, y) \in(A \times B) \cup(B \times A)
$$

so that $T: A \cup B \rightarrow A \cup B$ is asymptotically nonexpansive. If $x \in A$ is a best proximity point of $A$ and $y \in B$ is a best proximity point of $B$, then $\lim _{n \rightarrow \infty} d\left(T^{n} x, T^{n} y\right)=D$ and $T^{2 n} x \rightarrow z_{x}=$ $z(x)$ and $T^{2 n} y \rightarrow z_{y}=z(y)$ which are best proximity points of $A$ and $B$ (not being necessarily identical to $x$ and $y$ ), respectively, if furthermore, $T: A \cup B \rightarrow A \cup B$ is continuous.

(ii) Property (i) also holds if $T: A \cup B \rightarrow A \cup B$ satisfies (3.1) subject to (3.2), (3.22), (3.23)(3.24) and (3.5b) with $d\left(T^{n} x, T^{n} y\right) \geq d(x, y) ; \forall(x, y) \in(A \cup B) \times(B \cup A)$.

(iii) Assume that $T: A \cup B \rightarrow A \cup B$ is asymptotically $\beta$-strictly pseudocontractive in the intermediate sense so that (3.21a)-(3.21b) holds with $0 \leq \beta_{n}=\beta<1,\left\{\mu_{n}\right\} \subset\left[-1, \frac{1-\beta}{2 \beta}\right)$, 
$\left\{\alpha_{n}\right\} \subset[1, \infty), \alpha_{n} \rightarrow 1$ and $\mu_{n} \rightarrow-1$, as $n \rightarrow \infty ; \forall x, y \in A, \forall n \in \mathbf{N}$. Then $T: A \cup B \rightarrow A \cup B$ is asymptotically nonexpansive and Property (i) holds.

(iv) $T: A \cup B \rightarrow A \cup B$ is asymptotically pseudocontractive in the intermediate sense if (3.22) holds with $\left\{\beta_{n}\right\} \subset[0,1],\left\{\mu_{n}\right\} \subset\left[-1, \frac{1-\beta_{n}}{2 \beta_{n}}\right),\left\{\alpha_{n}\right\} \subset[1, \infty), \beta_{n} \rightarrow 1, \alpha_{n} \rightarrow 1$ and $\mu_{n} \rightarrow$ -1 as $n \rightarrow \infty ; \forall x, y \in A, \forall n \in \mathbf{N}$. Then $T: A \cup B \rightarrow A \cup B$ is asymptotically nonexpansive and Property (i) holds.

(v) If the conditions of Property (iv) are modified as $\alpha_{n} \in[0, \infty), \mu_{n} \in\left[-1, \frac{1-\beta}{2 \beta}\right), \mu_{n} \rightarrow$ $\mu \in\left[-1, \frac{1-\alpha-2 \beta}{2 \beta}\right) ; \forall n \in \mathbf{N}, \alpha_{n} \rightarrow \alpha \in[0,1)$ as $n \rightarrow \infty$ and $\beta_{n}=\beta \in[0,1)$ in (3.22), then $T: A \cup B \rightarrow A \cup B$ is asymptotically $\beta$-strictly contractive in the intermediate sense. Also, $T: A \cup B \rightarrow A \cup B$ has a unique best proximity point $z$ in $A$ and a unique best proximity point $T z$ in $B$ to which the sequences $\left\{T^{2 n} x\right\}$ and $\left\{T^{2 n+1} x\right\}$ converge; $\forall x \in A$. If $x \in B$, then $T^{2 n} x \rightarrow T z$ and $T^{2 n+1} x \rightarrow z$ as $n \rightarrow \infty$.

(vi) If (3.4) is modified by $\alpha_{n} \in[0, \infty),\left\{\beta_{n}\right\} \subset[0,1), \mu_{n} \in\left[-1, \frac{1-\beta_{n}}{2 \beta_{n}}\right), \mu_{n} \rightarrow \mu \in\left[-1,-\frac{1+\alpha}{2}\right)$; $\forall n \in \mathbf{N}, \alpha_{n} \rightarrow \alpha \in[0,1)$ and $\beta_{n} \rightarrow 1$ as $n \rightarrow \infty$, then $T: A \cup B \rightarrow A \cup B$ is asymptoticallystrictly contractive in the intermediate sense. Also, $T: A \cup B \rightarrow A \cup B$ has a unique best proximity point in $A$ and a unique best proximity point in $B$ to which the sequences $\left\{T^{2 n} x\right\}$ and $\left\{T^{2 n+1} x\right\}$ converge as in Property (v).

Proof The second condition of (2.18) now becomes under (3.1)-(3.2), or (3.3)-(3.4), and (3.23)-(3.24)

$$
\begin{aligned}
& d\left(T^{n} x, T^{n} y\right)^{2} \leq k_{b n} d^{2}(x, y)+\frac{\xi_{n}+\gamma_{n} D^{2}}{1-\beta_{n}\left(1+2 \mu_{n}\right)} \\
& \quad \Rightarrow \quad \exists \lim _{n \rightarrow \infty} d\left(T^{n} x, T^{n} y\right) \in[D, d(x, y)] ; \quad \forall(x, y) \in(A \cup B) \times(B \cup A)
\end{aligned}
$$

since $k_{n} \rightarrow 1$ and $\gamma_{n}(x, y) \rightarrow\left(1-k_{n}\right) \rightarrow 0$ as $n \rightarrow \infty ; \forall(x, y) \in(A \cup B) \times(B \cup A)$. Also, if $T$ : $A \cup B \rightarrow A \cup B$ is continuous, then $\lim _{n \rightarrow \infty} d\left(T^{2 n} x, T^{2 n} y\right)=d\left(\lim _{n \rightarrow \infty} T^{2 n} x, \lim _{n \rightarrow \infty} T^{2 n} \times\right.$ $y)=D$ so that $T^{2 n} x \in A, \lim _{n \rightarrow \infty} T^{2 n} x \in A, T^{2 n} y \in B$ and $\lim _{n \rightarrow \infty} T^{2 n} x \in B$ since $A$ and $B$ are closed and $T(A) \subseteq B$ and $T(B) \subseteq A$. This proves Properties (i)-(ii). To prove Property (iii), note that if $T: A \cup B \rightarrow A \cup B$ is asymptotically $\beta$-strictly pseudocontractive in the intermediate sense under (3.21a)-(3.21b)-(3.23) with $0<\beta_{n}=\beta<1 ; \forall n \in \mathbf{N}$, $1 \leq \alpha_{n} \rightarrow 1$ as $n \rightarrow \infty$ and (3.22) holds for $\mu_{n} \rightarrow-1$ as $n \rightarrow \infty$, then $T: A \cup B \rightarrow A \cup B$ is asymptotically nonexpansive and $D \leq d\left(T^{n} x, T^{n} y\right) \leq d(x, y)$ as $n \rightarrow \infty$ with $d(x, y)=D$ if $x \in A$ and $y \in B$ are best proximity points. Also, $\exists \lim _{n \rightarrow \infty} d\left(T^{n} x, T^{n} y\right) \in[D, d(x, y)]$; $\forall(x, y) \in(A \times B) \cup(B \times A)$ and $T^{2 n} x \in A, \lim _{n \rightarrow \infty} T^{2 n} x \in A, T^{2 n} y \in B$ and $\lim _{n \rightarrow \infty} T^{2 n} x \in B$ if $T: A \cup B \rightarrow A \cup B$ is continuous. Then Property (i) holds. Property (iv) is proven in a similar way as (iii) since $T: A \cup B \rightarrow A \cup B$ is again asymptotically nonexpansive. Properties (v)-(vi) follow since in both cases $T: A \cup B \rightarrow A \cup B$ becomes a cyclic strictly contractive self-mapping for all $n(\in \mathbf{N}) \geq n_{0}$ with $k_{j n}<1 ; \forall j, n\left(\geq n_{0}\right) \in \mathbf{N}$ and some finite $n_{0} \in \mathbf{N}$ in Theorem 3.3, Eq. (3.14). Thus, it is a direct proof that $\exists \lim _{n \rightarrow \infty} d\left(T^{2 n} x, T^{2 n} y\right)=D$; $\forall(x, y) \in A \times B \cup B \times A$ with $\left\{T^{2 n} x\right\} \subset A$ and $\left\{T^{2 n} y\right\} \subset B$ if $x \in A$ and $y \in B$ since $T(A) \subseteq B$ and $T(B) \subseteq A$. Also, $\exists \lim _{n \rightarrow \infty} d\left(T^{2 n} x, T^{2 n+2} x\right)=0 ; \forall x \in A \cup B$. Furthermore, $T^{2 n} x \rightarrow z$ and $T^{2 n} y \rightarrow T z ; \forall x \in A, \forall y \in B$ and there are unique best proximity points $z \in A$ and $T z \in B$. The convergence of the iterations to unique best proximity points follows using similar arguments as those used in the proof of Theorem 3.3(ii) based on the uniform convexity of the complete metric space and the fact that the subsets $A$ and $B$ are nonempty, convex and closed. 
Remark 3.6 Note that the existence of Theorem 3.5 of $x \in A$ and $y \in B$ such that $D=$ $d(x, y)$ is guaranteed if $A$ is nonempty, bounded, closed and convex and $B$ is nonempty closed and convex is also guaranteed if $A$ is compact and $B$ is approximately compact with respect to $A$, i.e., if every sequence $\left\{x_{n}\right\} \subset B$, such that $d\left(y, x_{n}\right) \rightarrow d(y, B)$ for some $y \in A$, has a convergent subsequence $[6,7,31]$.

Example 3.7 Consider the time-varying scalar controlled discrete dynamic system:

$$
x_{k+1}=T x_{k} \equiv F_{1}\left(x_{k}\right)=G\left(x_{k}\right) x_{k}+u_{k}\left(x_{k}\right)=\left(G\left(x_{k}\right)+K_{k}\left(x_{k}\right)\right) x_{k} ; \quad x_{1} \in \mathbf{R}, \forall k \in \mathbf{N}
$$

under the feedback control sequence

$$
\begin{aligned}
u_{k}= & u_{k}\left(x_{k}\right)=K_{k}=K_{k}\left(x_{k}\right)=F_{1}\left(x_{k}\right)-G\left(x_{k}\right) x_{k} ; \quad \forall k \in \mathbf{N} \\
= & \frac{\alpha_{1}\left(x_{k-n+1}\right)+\beta_{1}\left(x_{k-n+1}\right)}{1-\beta_{1}\left(x_{k-n+1}\right)\left(1+2 \mu_{1}\left(x_{k-n+1}\right)\right)}\left(\prod_{j=0}^{n-2}\left[F_{1}\left(x_{k+j-n+1}\left(x_{k-n+1}\right)\right)\right]\right)^{-1} \\
& -G\left(x_{k}\right) x_{k} ; \quad \forall k \in \mathbf{N}_{0}
\end{aligned}
$$

so that

$$
x_{k+n}=F_{n}\left(x_{k}\right) x_{k} ; \quad x_{1} \in \mathbf{R} ; \forall k \in \mathbf{N},
$$

where $F_{n}\left(x_{k}\right)=\prod_{j=0}^{n-1}\left[F_{1}\left(x_{k+j}\left(x_{k}\right)\right)\right]=F_{1}\left(x_{k+n-1}\left(x_{k}\right)\right)\left(\prod_{j=0}^{n-2}\left[F_{1}\left(x_{k+j}\left(x_{k}\right)\right)\right]\right) ; \forall k, n \in \mathbf{N}$ for some given nonempty bounded set $A_{0}$, where $\left\{u_{k}\left(x_{k}\right)\right\}$ is the control sequence. The above model can describe discrete-time dynamic systems under time-varying sampling periods or under a time-varying parameterization in general [39]. Assume that the suitable controlled solution (3.28) is of the form

$$
\left|F_{n}\left(x_{k}\right)\right|=\frac{\alpha_{n}\left(x_{k}\right)+\beta_{n}\left(x_{k}\right)}{1-\beta_{n}\left(x_{k}\right)\left(1+2 \mu_{n}\left(x_{k}\right)\right)} ; \quad \forall k \in \mathbf{N} .
$$

Then

$$
\begin{aligned}
\left|F_{n}\right| & =\left|F_{n}\left(x_{k}\right)\right|=k_{n}^{2}=k_{n}^{2}\left(x_{k}\right)=\frac{\alpha_{n}\left(x_{k}\right)+\beta_{n}\left(x_{k}\right)}{1-\beta_{n}\left(x_{k}\right)\left(1+2 \mu_{n}\left(x_{k}\right)\right)} \\
& =F_{1}\left(x_{k+n-1}\left(x_{k}\right)\right)\left(\prod_{j=0}^{n-2}\left[F_{1}\left(x_{k+j}\left(x_{k}\right)\right)\right]\right) ; \quad \forall k \in \mathbf{N} \\
& =\frac{\alpha_{1}\left(x_{k-n+1}\right)+\beta_{1}\left(x_{k-n+1}\right)}{1-\beta_{1}\left(x_{k-n+1}\right)\left(1+2 \mu_{1}\left(x_{k-n+1}\right)\right)}\left(\prod_{j=0}^{n-2}\left[k_{1}^{2}\left(x_{k+j}\left(x_{k}\right)\right)\right]\right) ; \quad \forall k \in \mathbf{N} \\
& =\prod_{j=0}^{n-1}\left[u_{k+j}\left(x_{k}\right)+G\left(x_{k}\right) x_{k}\right] \\
& =\left(u_{k+n-1}\left(x_{k}\right)+G\left(x_{k+n-1}\right) x_{k+n-1}\right)\left(\prod_{j=0}^{n-2}\left[u_{k+j}\left(x_{k}\right)+G\left(x_{k+k}\right) x_{k+j}\right]\right) ; \quad \forall k \in \mathbf{N} .
\end{aligned}
$$


The identities (3.30) allow the feedback generation of the control sequence (3.26) from its previous values and previous solution values as follows:

$$
\begin{aligned}
u_{k+n-1}\left(x_{k}\right) & =\frac{\alpha_{n}\left(x_{k}\right)+\beta_{n}\left(x_{k}\right)}{1-\beta_{n}\left(x_{k}\right)\left(1+2 \mu_{n}\left(x_{k}\right)\right)}\left(\prod_{j=0}^{n-2}\left[u_{k+j}\left(x_{k}\right)+G\left(x_{k+k}\right) x_{k+j}\right]\right)^{-1}-G\left(x_{k+n-1}\right) x_{k+n-1} \\
& =F_{1}\left(x_{k+n-1}\right)-G\left(x_{k+n-1}\right) x_{k+n-1} ; \quad \forall k \in \mathbf{N}
\end{aligned}
$$

for given parameterizing scalar sequences which can be dependent on the state $x_{k}$ (see Example 2.9). We are now defining a cyclic self-map $T:[D / 2, \infty) \cup(-\infty, D / 2] \rightarrow[D / 2, \infty) \cup$ $(-\infty, D / 2]$ so that the solution belongs alternately to positive (respectively, nonnegative) and negative (respectively, nonpositive) real intervals $[D / 2, \infty)$ and $(-\infty, D / 2]$ if $D:=$ $\operatorname{dist}([D / 2, \infty),(-\infty, D / 2])>0$ (respectively, if $D=0)$, that is, $T([D / 2, \infty)) \subseteq(-\infty, D / 2]$ and $T((-\infty, D / 2]) \subseteq[D / 2, \infty)$. For such an objective, consider the scalar bounded sequences $\left\{\lambda_{n k}\left(x_{k}\right)\right\},\left\{\alpha_{n k}^{0}\left(x_{k}\right)\right\}$ and $\left\{\beta_{n k}^{0}\left(x_{k}\right)\right\}$ such that $\lambda_{n k}=\lambda_{n k}\left(x_{k}\right), \alpha_{n k}^{0}=\alpha_{n k}^{0}\left(x_{k}\right)$ and $\beta_{n k}^{0}=\beta_{n k}^{0}\left(x_{k}\right)$; $\forall k \in \mathbf{N}_{0}, \forall n \in \mathbf{N}$ which satisfy

$$
\begin{aligned}
& \alpha_{n k}=\lambda_{n} \alpha_{n k}^{0} ; \quad \beta_{n k}=\lambda_{n} \beta_{n k}^{0} ; \quad \lambda_{n k} \geq \frac{1}{2}\left(\frac{\alpha_{n k}^{0}+\beta_{n k}^{0}}{1-\beta_{n k}\left(1+2 \mu_{n k}\right)}\right)^{-1} D, \\
& \min \left(\alpha_{n k}^{0}+\beta_{n k}^{0}\right)>0 ; \quad \forall k, n \in \mathbf{N}, \\
& \exists \lim _{n \rightarrow \infty} \lambda_{n k}=\frac{1}{2} D \lim _{n \rightarrow \infty}\left(\frac{\alpha_{n k}^{0}+\beta_{n k}^{0}}{1-\beta_{n k}\left(1+2 \mu_{n k}\right)}\right)^{-1}=\lambda ; \quad \forall k \in \mathbf{N} .
\end{aligned}
$$

Note that by using the Euclidean distance and norm on $\mathbf{R}$, it is possible to apply the theoretical formalism to the expressions $D / 2 \leq\left\|x_{k+n}\right\|=d\left(T^{n} x_{k}, 0\right) \leq\left\|F_{n}\right\| d\left(x_{k}, 0\right) ; \forall k, n \in \mathbf{N}$ to prove convergence to the best proximity points $\pm D / 2$ to which the sequences $\left\{T^{2 n} x_{1}\right\}$ and $\left\{T^{2 n+1} x_{1}\right\}$ converge, respectively if $x_{1} \in[D / 2, \infty)$ and conversely if $x_{1} \in(-\infty, D / 2]$. Assume that:

(1) The constraints (3.32a)-(3.32b) hold;

(2) The parametrical constraints of the various parts (a) to (d) of Example 2.9 hold with the replacements and its appropriate replacements of the constraints $\alpha_{n k}$ (Example 2.9) $\rightarrow$ $\alpha_{n k}^{0}$ (Example 3.7), $\beta_{n k}$ (Example 2.9) $\rightarrow \beta_{n k}^{0}$ (Example 3.7);

(3) $\left\{\alpha_{n k}\right\}$ and $\left\{\beta_{n k}\right\}$ are redefined for this example from $\left\{\alpha_{n k}^{0}\right\}$ and $\left\{\beta_{n k}^{0}\right\}$, respectively, from (3.32a)-(3.32b).

From Theorem 3.5, the various properties of Example 2.9 hold also for this example if $D=0$ so that the cyclic self-map is such that it alternates the values of the solution sequence between $\mathbf{R}_{0+}$ and $\mathbf{R}_{0-}$. The unique fixed point to which the solution converges is $\{0\}$. If $D>0$, then the corresponding results are modified by convergence to each of the unique best proximity points to which the sequences $\left\{T^{2 n} x_{1}\right\}$ and $\left\{T^{2 n+1} x_{1}\right\}$ converge; $\forall x_{1} \in[D / 2, \infty) \cup(-\infty, D / 2]$. 


\section{Acknowledgements}

The author is very grateful to the Spanish Government for its support of this research through Grant DPI2012-30651, and to the Basque Government for its support of this research through Grants IT378-10 and SAIOTEK S-PE12UN015. He is also grateful to the University of Basque Country for its financial support through Grant UFI 2011/07 and to the referees for their useful comments.

\section{Received: 17 September 2012 Accepted: 17 May 2013 Published: 5 June 2013}

\section{References}

1. Sahu, DR, Xu, HK, Yao, JC: Asymptotically strict pseudocontractive mappings in the intermediate sense. Nonlinear Anal., Theory Methods Appl. 70, 3502-3511 (2009)

2. Qin, X, Kim, JK, Wang, T: On the convergence of implicit iterative processes for asymptotically pseudocontractive mappings in the intermediate sense. Abstr. Appl. Anal. 2011, Article ID 468716 (2011). doi:10.1155/2011/468716

3. Ceng, LC, Petrusel, A, Yao, JC: Iterative approximation of fixed points for asymptotically strict pseudocontractive type mappings in the intermediate sense. Taiwan. J. Math. 15(2), 587-606 (2011)

4. Duan, $\mathrm{P}, \mathrm{Zhao}, \mathrm{J}$ : Strong convergence theorems for system of equilibrium problems and asymptotically strict pseudocontractions in the intermediate sense. Fixed Point Theory Appl. 2011, 13 (2011). doi:10.1186/1687-1812-2011-13

5. Kirk, WA, Srinivasan, PS, Veeramani, P: Fixed points for mappings satisfying cyclical contractive conditions. Fixed Point Theory 4(1), 79-89 (2003)

6. Eldred, AA, Veeramani, P: Existence and convergence of best proximity points. J. Math. Anal. Appl. 323, 1001-1006 (2006)

7. Karpagam, S, Agrawal, S: Best proximity point theorems for $p$-cyclic Meir-Keeler contractions. Fixed Point Theory Appl. 2009, Article ID 197308 (2009). doi:10.1155/2009/197308

8. Di Bari, C, Suzuki, T, Vetro, C: Best proximity points for cyclic Meir-Keeler contractions. Nonlinear Anal., Theory Methods Appl. 69(11), 3790-3794 (2008)

9. De la Sen, M: Linking contractive self-mappings and cyclic Meir-Keeler contractions with Kannan self-mappings. Fixed Point Theory Appl. 2010, Article ID 572057 (2010). doi:10.1155/2010/572057

10. De la Sen, M: Some combined relations between contractive mappings, Kannan mappings reasonable expansive mappings and T-stability. Fixed Point Theory Appl. 2009, Article ID 815637 (2009). doi:10.1155/2009/815637

11. Suzuki, T: Some notes on Meir-Keeler contractions and L-functions. Bull. Kyushu Inst. Technol. 53, 12-13 (2006)

12. Derafshpour, M, Rezapour, S, Shahzad, N: On the existence of best proximity points of cyclic contractions. Adv. Dyn. Syst. Appl. 6(1), 33-40 (2011)

13. Rezapour, S, Derafshpour, M, Shahzad, N: Best proximity points of cyclic $\varphi$-contractions on reflexive Banach spaces. Fixed Point Theory Appl. 2010, Article ID 946178 (2010). doi:10.1155/2010/946178

14. Al-Thagafi, MA, Shahzad, N: Convergence and existence results for best proximity points. Nonlinear Anal., Theory Methods Appl. 70(10), 3665-3671 (2009)

15. Rus, IA: Cyclic representations and fixed points. Ann. T. Popoviciu Semin. Funct. Equ. Approx. Convexity 3, 171-178 (2005)

16. Pacurar, M, Rus, IA: Fixed point theory for cyclic $\varphi$-contraction. Nonlinear Anal., Theory Methods Appl. 72(3-4), 1181-1187 (2010)

17. Karapinar, E: Fixed point theory for cyclic weak $\phi$-contraction. Appl. Math. Lett. 24, 822-825 (2011)

18. Shazhad, N, Sadiq Basha, S, Jeyaraj, R: Common best proximity points: global optimal solutions. J. Optim. Theory Appl. 148(1), 69-78 (2011)

19. Vetro, C: Best proximity points: convergence and existence theorems for $p$-cyclic mappings. Nonlinear Anal., Theory Methods Appl. 73(7), 2283-2291 (2010)

20. De la Sen, M: On a general contractive condition for cyclic self-mappings. J. Appl. Math. 2011, Article ID 542941 (2011). doi:10.1155/2011/542941

21. Yao, YH, Liu, YC, Chen, CP: Algorithms construction for nonexpansive mappings and inverse-strongly monotone mappings. Taiwan. J. Math. 15(5), 1979-1998 (2011)

22. Yao, YH, Chen, RD: Regularized algorithms for hierarchical fixed-point problems. Nonlinear Anal., Theory Methods Appl. 74(17), 6826-6834 (2011)

23. Qin, X, Kang, SM, Agarwal, RP: On the convergence of an implicit iterative process for generalized asymptotically quasi-nonexpansive mappings. Fixed Point Theory Appl. 2010, Article ID 714860 (2010). doi:10.1155/2010/714860

24. Pathak, HK, Khan, MS, Tiwari, R: A common fixed point theorem and its application to nonlinear integral equations. Comput. Math. Appl. 53(6), 961-971 (2007). doi:10.1016/j.camwa.2006.08.046

25. Khan, MS, Nashine, HK: On invariant approximation for noncommutative mappings in locally convex spaces. J. Comput. Anal. Appl. 10(1), 7-15 (2008)

26. Nashine, HK, Khan, MS: An application of fixed point theorem to best approximation in locally convex space. Appl. Math. Lett. 23(2), 121-127 (2010). doi:10.1016/j.aml.2009.06.025

27. Nashine, HK, Khan, MS: Common fixed points versus invariant approximation in nonconvex sets. Appl. Math. E-Notes 9, 72-79 (2009)

28. Pathak, HK, Tiwari, R, Khan, MS: A common fixed point theorem satisfying integral type implicit relations. Appl. Math. E-Notes 7, 222-228 (2007)

29. De la Sen, M, Agarwal, RP: Fixed point-type results for a class of extended cyclic self-mappings under three general weak contractive conditions of rational type. Fixed Point Theory Appl. 2012, 102 (2012). doi:10.1186/1687-1812-2011-102

30. De la Sen, M, Agarwal, RP: Some fixed point-type results for a class of extended cyclic self-mappings with a more general contractive condition. Fixed Point Theory Appl. 2011, 59 (2011). doi:10.1186/1687-1812-2011-59

31. Basha, SS, Shahzad, N: Best proximity point theorems for generalized proximal contractions. Fixed Point Theory Appl. 2012, 42 (2012). doi:10.1186/1687-1812-2012-42

32. Chen, CM, Lin, CJ: Best periodic proximity point theorems for cyclic weaker Meir-Keeler contractions. J. Appl. Math. 2012, Article ID 856974 (2012). doi:10.1155/2012/856974 
33. Caballero, J, Harjani, J, Sadarangani, K: A best proximity point theorem for Geraghty-contractions. Fixed Point Theory Appl. 2012, 231 (2012)

34. Mongkolkeha, C, Cho, YJ, Kuman, P: Best proximity points for generalized proximal C-contraction mappings in metric spaces with partial orders. J. Inequal. Appl. 2013, 94 (2013)

35. Karapinar, E: Best proximity points of Kannan type cyclic weak $\phi$-contractions in ordered metric spaces. An. Stiint. Univ. Ovidius Constanta, Ser. Mat. 20(3), 51-63 (2012)

36. Karapinar, E: Best proximity points of cyclic mappings. Appl. Math. Lett. 25(11), 1761-1766 (2012)

37. Karapinar, E, Erhan, IM: Best proximity points on different type contractions. Appl. Math. Inf. Sci. 5(3), $558-569$ (2011)

38. Raj, VS: A best proximity theorem for weakly contractive non-self mappings. Nonlinear Anal., Theory Methods Appl. 74(14), 4804-4808 (2011)

39. De la Sen, M: Application of the nonperiodic sampling to the identifiability and model-matching problems in dynamic systems. Int. J. Syst. Sci. 14(4), 367-383 (1983)

doi:10.1186/1687-1812-2013-146

Cite this article as: De la Sen: On best proximity points for pseudocontractions in the intermediate sense for non-cyclic and cyclic self-mappings in metric spaces. Fixed Point Theory and Applications 2013 2013:146.

\section{Submit your manuscript to a SpringerOpen ${ }^{\circ}$ journal and benefit from:}

- Convenient online submission

- Rigorous peer review

- Immediate publication on acceptance

Open access: articles freely available online

- High visibility within the field

- Retaining the copyright to your article 